\title{
ARTICLE
}

\section{Entamoeba histolytica-induced IL-1 $\beta$ secretion is dependent on caspase-4 and gasdermin D}

\author{
Jeanie Quach ${ }^{1}$, France Moreau ${ }^{1}$, Christina Sandall ${ }^{1}$ and Kris Chadee ${ }^{1}$
}

During invasion, Entamoeba histolytica $(E h)$ encounter macrophages and activate them to elicit tissue damaging pro-inflammatory responses. When $E h$ binds macrophages via the Gal-lectin, surface EhCP-A5 RGD sequence ligates $\alpha_{5} \beta_{1}$ integrin to activate caspase1 in a complex known as the NLRP3 inflammasome. In this study, we investigated Eh requirements underlying macrophage caspase- 4 and -1 activation and the role caspase- 4 and gasdermin D (GSDMD) play in augmenting pro-inflammatory cytokine responses. Caspase- 4 activation was similar to caspase- 1 requiring live Eh attachment via the Gal-lectin and EhCP-A5. However, unlike caspase-1, caspase- 4 activation was independent of ASC and NLRP3. Using CRISPR/Cas9 gene editing of caspase-4 and -1 and GSDMD, we determined that caspase- 1 and bioactive IL-1 $\beta$ release was highly dependent on caspase- 4 activation and cleavage of GSDMD in response to $E$. Formaldehyde cross-linking to stabilize protein-protein interactions in transfected COS-7 cells stimulated with Eh revealed that caspase-4 specifically interacted with caspase-1 in a protein complex that enhanced the cleavage of caspase-1 CARD domains to augment IL-1 $\beta$ release. Activated caspase- 4 and -1 cleaved GSDMD liberating the $\mathrm{N}$-terminal $\mathrm{p} 30$ pore-forming fragment that caused the secretion of IL-1 $\beta$. These findings reveal a novel role for caspase- 4 as a sensor molecule to amplify proinflammatory responses when macrophage encounters $E h$.

Mucosal Immunology (2019) 12:323-339; https://doi.org/10.1038/s41385-018-0101-9

\section{INTRODUCTION}

Entamoeba histolytica (Eh) is an extracellular protozoan parasite that colonizes the large intestine of humans. In $90 \%$ of cases the parasite causes asymptomatic infection, while in 10\% of individuals $E h$ invades the colonic mucosal barrier and triggers an acute pro-inflammatory response. This parasite annually causes 100 million cases of amebiasis leading to 100,000 deaths. ${ }^{1}$ Manifestations of the disease include amebic colitis and the potential to cause fatality due to liver, lung, or brain abscesses. ${ }^{2}$ Although there are more cases of amebiasis in developing countries with poor sanitation and lack of clean water, host factors also contribute to the susceptibility of disease. These factors include host genetics and malnutrition. ${ }^{3,4}$ When Eh breaches innate host defenses and is sensed by the immune system, an appropriate pro-inflammatory response is triggered to eliminate the parasite, but at the same time it can be damaging to innocent bystander cells. Hence, the inflammatory response is tightly regulated. One of the first cells that $E h$ encounters within the innate immune compartment is resident macrophage, which is highly phagocytic and bactericidal. Macrophages are major producers of interleukin (IL)- $1 \beta$ and tumor necrosis factor (TNF)- $\alpha$, the hallmark cytokines in amebiasis known to be important in recruiting immune cells to the site of infection. This response can be protective as interferon (IFN) $-\gamma$ and TNF- $\alpha$ act synergistically to activate macrophages to release nitric oxide that can kill up to $87 \%$ of Eh in vitro. ${ }^{5}$ This dynamic Eh-macrophage interaction is therefore critical for understanding host defense and disease pathogenesis.

Another key determinant of disease outcome are the virulence factors expressed by $E h$. One of the most well-characterized virulence factor of $E h$ is the Gal/GalNAc lectin (Gal-lectin) important for mediating attachment to host cells via galactose and $\mathrm{N}$-acetylgalactosamine residues. ${ }^{6,7}$ We recently showed that both Eh cysteine protease (EhCP)-A1 and EhCP-A4 activate caspase-6-dependent cleavage of the cytoskeletal proteins talin, Pyk2 and paxillin in macrophages to trigger downstream inflammatory signaling pathways. ${ }^{8}$ Innate sensing of Eh also occurs through high molecular weight complexes known as inflammasomes, which are intracellular sensors made up of domains including the NLRP3 sensor, ASC adapter, and caspase-1. When Eh contacts macrophages with the Gal-lectin it forms an intercellular bridge allowing EhCP-A5 RGD sequence to bind $a_{5} \beta_{1}$ integrin to activate the NLRP3 inflammasome. ${ }^{9,10}$ While it is known that caspase-1 activation is important in regulating the secretion of IL- $1 \beta$ and IL- $18,{ }^{11}$ the role caspase- 4 play to the overall pro-inflammatory response is less characterized. Caspase- 4 is important in inflammation by regulating IL-1a, IL-18, IL8 , and MIP-1 secretions and cell death. ${ }^{12-14}$ Studies drawn from the murine ortholog, caspase-11, show that caspase-11 can activate caspase-1. ${ }^{15,16}$ Gasdermin D (GSDMD) was recently identified as the critical mediator for pyroptotic cell death initiated by caspase-1, -4 , and -11 activation. ${ }^{17-19}$ Inflammatory caspases (caspase-1/4/5/11) cleave GSDMD into a $31 \mathrm{kDa} \mathrm{N}$-terminal fragment and a $22 \mathrm{kDa} \mathrm{C}$ terminal fragment, but only the $\mathrm{N}$-terminal fragment form pores in the plasma membrane to elicit cell lysis. ${ }^{18,19}$ GSDMD-deficient cells did not impact caspase- 1 activation, but secretion of IL-1 $\beta$ was blocked indicating that GSDMD contributes to IL-1 $\beta$ secretion. ${ }^{17,18}$ Although $E h$ induces minimal macrophage cell death and robust IL$1 \beta$ secretion, the role GSDMD play in this process has not been explored.

In this study, we found that Eh Gal-lectin and EhCP-A5 were critical components involved in activating both inflammatory

\footnotetext{
${ }^{1}$ Department of Microbiology, Immunology and Infectious Diseases, Snyder Institute for Chronic Diseases, University of Calgary, Calgary, Alberta, Canada
} Correspondence: Kris Chadee (kchadee@ucalgary.ca)

Received: 4 May 2018 Revised: 21 September 2018 Accepted: 1 October 2018

Published online: 25 October 2018 
caspase- 4 and -1 by similar mechanisms. Caspase- 4 activation involved potassium $\left(\mathrm{K}^{+}\right)$efflux and the generation of reactive oxygen species (ROS) but was independent of NLRP3 and ASC activation. Eh-induced caspase-4 activation enhanced the cleavage of caspase-1 CARD domains and both caspase cleaved GSDMD to facilitate bioactive IL-1 $\beta$ release. This study unravels new concepts on how Eh-induced activation of inflammatory caspases in macrophages can converge onto one role to shape the magnitude of the host pro-inflammatory responses in disease pathogenesis.

\section{RESULTS}

E. histolytica induces the activation and secretion of caspase-4 We have recently shown that direct interaction between $E h$ and macrophages via the Gal-lectin and coupling of EhCP-A5 RGD motif to $a_{5} \beta_{1}$ integrin activates caspase- 1 that subsequently cleaves pro-IL-1 $\beta$ into its active form. ${ }^{9,10}$ As other inflammatory caspases are activated upon $E h$ contact $^{8}$ that can potentially regulate inflammasome activation and IL-1 $\beta$, in this study we determined the kinetics of $E$-induced caspase- 4 and -1 in human THP-1 macrophages. Eh activated caspase-4 (by the appearance of the intermediate forms) in a dose- and time-dependent manner with 1:20 Eh to macrophage ratio being the sub-optimal dosage (Figs 1a, c). For comparison, caspase-1 activation as indicated by the cleavage of the $\mathrm{N}$-terminal CARD protein ( $\mathrm{p} 10$ and $\mathrm{p} 11$ doublet bands) that reflects processing and activation is shown for all studies. Similarly, cleaved IL-1 $\beta(17 \mathrm{kDa})$ protein was also immunoblotted to demonstrate conversion to bioactive protein. In control macrophages (-ve) basal levels of caspase-1 remained in its inactive form, however, following Eh stimulation it was recruited into a complex that cleaved the $\mathrm{N}$-terminal CARD domain, which resulted in the formation of a heterodimer of p10 and p20 subunits. Eh activated caspase- 4 as indicated by the 30-34 kDa triplet bands (Figs 1a, c) in both the supernatants (SN) and the lysates (LYS). We originally thought that the appearance of a band around $20 \mathrm{kDa}$ would be the large subunit of caspase- 4 or a band of $10 \mathrm{kDa}$ for the small subunit, but these did not appear consistently with the caspase-4 M029-3 antibody. Therefore, the intermediate forms were used as the main indicators of activation. The reason for this is the cysteine catalytic site is found on the large subunit and processing of the pro-form of caspase- 4 generates several different intermediate products (full blots using the caspase- 4 and -1 antibody and IL- $1 \beta$ antibody are shown in Supplementary Figures S1A and S1B). Bioactive IL-1 $\beta$ levels increased dose-dependently with an increase in Eh to macrophage ratio, as detected using HEK-Blue $\mathrm{e}^{\mathrm{TM}} \mathrm{IL}-1 \beta$ reporter cells and the measurement of secreted embryonic alkaline phosphatase (SEAP) production (Fig. 1b) and by IL-1 $\beta$ ELISA (Supplementary Figure S1C). Interestingly, intracellular caspase-4 was activated as early as $10 \mathrm{~min}$; however, secretions did not appear until $30 \mathrm{~min}$ (Fig. 1c). This suggested that between 10 and $30 \mathrm{~min}$, caspase- 4 was cleaved from its inactive form, performed its function, either acting on substrates and/or interacting with other proteins and was then immediately secreted outside. The caspase- 4 active intermediate forms accumulated in the supernatants over $60 \mathrm{~min}$. Only the secretions indicated an obvious difference among the treatment conditions, whereas the lysates displayed minimal differences. Therefore, the majority of conclusions are based on the secretions of caspases. Bioactive IL-1 $\beta$ levels increased time-dependently, showing robust levels at $60 \min (p<0.001)$ with Eh stimulation, indicating that the caspases are activated prior to IL-1 $\beta$ release (Fig. $1 \mathrm{~d}$ ). The release of both inflammatory caspases and IL-1 $\beta$ were not due to cell death. This was confirmed using a lactate dehydrogenase (LDH) assay, which showed $<1 \%$ cell death with Eh stimulation for $10 \mathrm{~min}$ (Fig. 1e). Even with prolonged exposure to $E h$ up to $60 \mathrm{~min}$, there was only $5 \%$ cell death compared to LPS and nigericin (LN) stimulation for $60 \mathrm{~min}$ that resulted in $70 \%$ cell death compared to controls $(p<0.001)$. As a positive control for these studies we used the well-described agonist for NLRP3 inflammasome activation, monosodium urate crystals (MSU). MSU is readily taken up by macrophages via phagocytosis that subsequently causes lysosomal damage and rupture, ${ }^{20}$ and via the noncanonical pathway requiring caspase- 4 to activate caspase- 1 and to secrete IL- $1 \beta$ and IL-18. ${ }^{21}$ As IL- $1 \beta$ in the immunoblots (Figs $1 \mathrm{a}, \mathrm{c}$ ) was an excellent indicator of $\mathrm{IL}-1 \beta$ release, the SEAP assay was not performed in subsequent studies involving characterizing of the parasite and host factors. These results show that both caspase- 4 and -1 are activated simultaneously and are secreted upon Eh stimulation.

Caspase-4 activation requires live $E h$, contact with macrophage via the Gal-lectin and EhCP-A5 and involves cellular perturbations

$E h$ is unique in that it is an extracellular parasite that requires Gallectin mediated contact with host cells to activate inflammatory signaling proteins to elicit cellular cytotoxicity. ${ }^{8,10}$ Similarly, we also found that live $E h$ was critical for activating both caspase- 4 and -1 in macrophages. Neither highly concentrated secreted protein from $E h$, equivalent amounts of whole $E h$ lysates, cytoplasmic components or membrane components were able to activate the inflammatory caspases (Fig. 2a). To determine the requirements for Eh-macrophage contact, $55 \mathrm{mM}$ exogenous galactose was used to competitively block $E h$ from binding via Gal or GaINAc residues on the macrophage surface. Galactose treatment completely inhibited caspase- 4 activation and secretion as compared to glucose, the osmotic control, which was the same as Eh treatment alone (Fig. 2b). IL-1 $\beta$ secretion also corresponded to caspase- 1 activation where there was complete abrogation with galactose treatment. These results support the notion that inflammatory caspase activation is tightly regulated and only while sensing live $E h$ does the host see it as a danger signal that requires immediate attention. Among $E h$ virulent factors, cysteine proteases have been shown to play major roles in Eh pathogenesis. ${ }^{22}$ EhCP-A5 is a major protease that is membrane-bound and secreted by Eh, which is involved in cleaving the C-terminus of MUC2 mucin to dissolve the protective mucus barrier. ${ }^{23,24}$ To determine if EhCP-A5 played a role in caspase-4 activation, Eh was incubated overnight with the irreversible cysteine protease inhibitor E-64 and it did not affect caspase-4 and -1 activation or IL-1 $\beta$ protein secretion (Fig. $2 \mathrm{c}$ ). Complete inhibition was seen with EhCP-A5 ${ }^{-}$with no detectable caspase-4 activation and secretion compared to parasites carrying the empty vector EhAP$A^{-}$. These results show that adherence to macrophages through the Gal-lectin adhesin and EhCP-A5 is important for activating caspase-4.

Since the triggers that result in caspase- 1 activation were reported previously to involve $\mathrm{K}^{+}$efflux and ROS production, ${ }^{9}$ we were curious to know whether the same phenomenon occurred during the activation of caspase- 4 . Predictably, using increasing concentrations of $\mathrm{K}^{+}$added to macrophages for $1 \mathrm{~h}$ prior to $E h$ stimulation to block $\mathrm{K}^{+}$efflux led to a dose-dependent inhibition of caspase- 4 and -1 activation and IL-1 $\beta$ release (Fig. $2 d$ ). Compared to the osmotic control $\mathrm{NaCl}$ in equimolar amounts, only $\mathrm{KCl}$ was able to inhibit caspase-4 and -1 activation, demonstrating specificity of blocking $\mathrm{K}^{+}$channels (Supplementary Figure S1E). Furthermore, using increasing concentrations of diphenyleneiodonium chloride (DPI) dose-dependently inhibited caspase -4 and -1 activation and IL-1 $\beta$ release indicating that ROS is also a cellular event that triggers caspase activation (Fig. 2e). These results indicate that cellular stresses converge both to trigger caspase- 4 and -1 activation, which suggests that they are upstream of the pathway. To determine if $E h$-induced ATP release activated caspase- 4 in an autocrine fashion similarly to caspase- $1^{9}$, a $\mathrm{P} 2 \mathrm{X}_{7} \mathrm{R}$ inhibitor, oxidized ATP was used and it effectively inhibited caspase- 4 and -1 activation (Fig. 2f). 
a

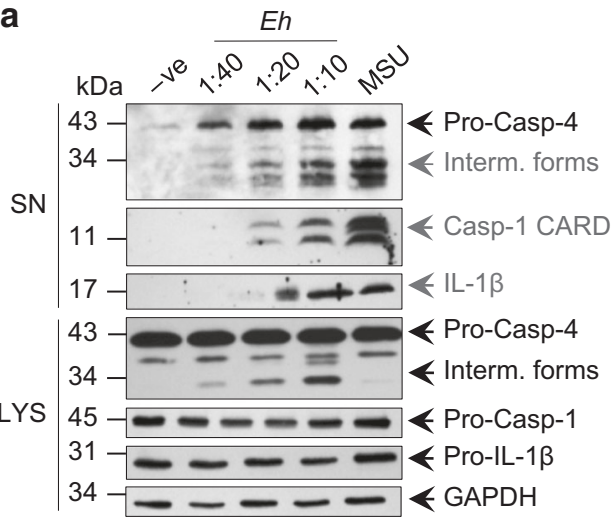

C

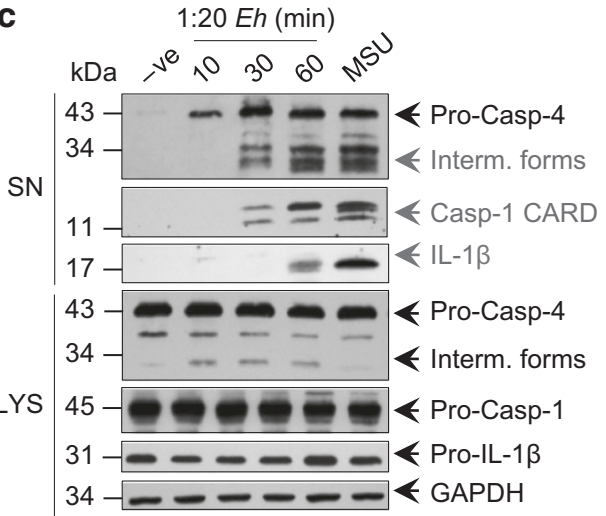

e

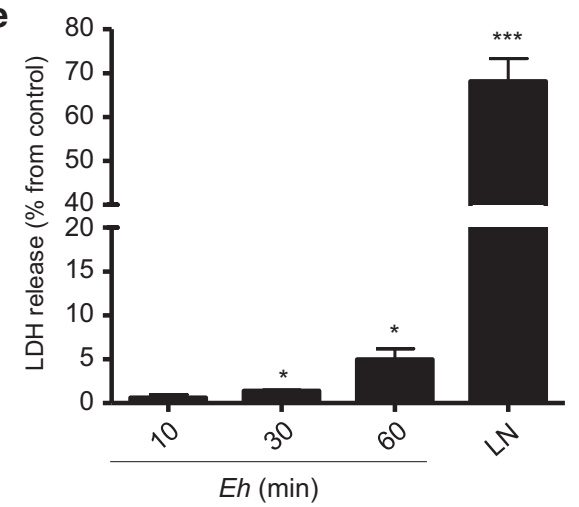

b

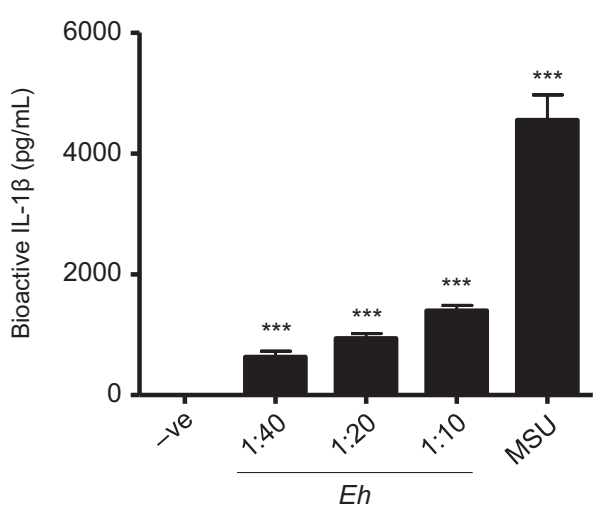

d

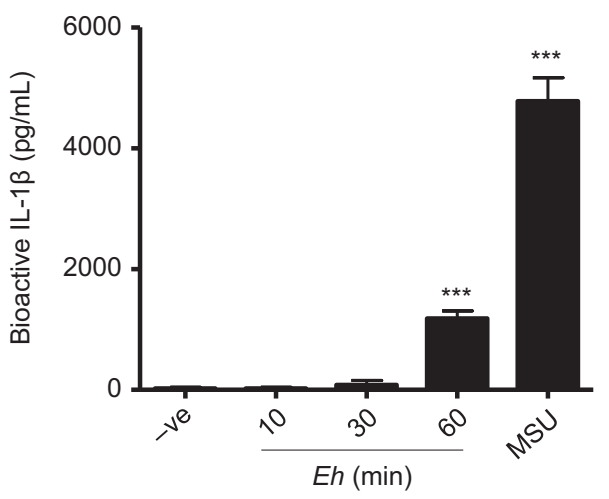

Fig. 1 E. histolytica activates caspase- 4 and -1 in a dose and time-dependently manner. a, b THP-1 macrophages were incubated with increasing ratios of $E h$ for $60 \mathrm{~min}$, and monosodium urate crystals (MSU) were added at $300 \mu \mathrm{g} / \mathrm{mL}$ for $6 \mathrm{~h}$ as a positive control. Cells only were used as an internal control. Cell supernatant was TCA precipitated and cells were washed and lysed. Equal amounts of lysates were loaded onto SDS-PAGE gel and immunoblotted with the indicated antibodies. $\mathbf{b}$ Cell supernatant was added to HEK-Blue ${ }^{\mathrm{TM}}$ IL-1 $\beta$ reporter cells to detect bioactive IL-1 $\beta$ using the SEAP assay. c-e THP-1 macrophages were incubated for increasing amounts of time with 1:20 Eh to macrophage ratio. Immunoblot analysis was performed for caspase-4, caspase-1, IL-1 $\beta$ in both the supernatants (SN) and lysates (LYS), and blots were reprobed for GAPDH. d Cell supernatant was added to HEK-Blue ${ }^{\mathrm{TM}}$ IL-1 $\beta$ reporter cells to detect bioactive IL-1 $\beta$ using the SEAP assay. e Cell death was measured by lactate dehydrogenase (LDH) release into the culture supernatant and is shown as a percentage of LDH release compared to non-stimulated cells (control). LN is abbreviated for LPS ( $50 \mathrm{ng} / \mathrm{mL})$ and nigericin (10 $\mu$ M) stimulation for 60 min. Data are representative of three independent experiments and statistical significance was calculated with an ANOVA and Bonferroni's post-hoc test $\left({ }^{*} p<0.05,{ }^{* * *} p<0.001\right)$. Bars represent \pm SEM

E. histolytica-induced caspase- 4 and -11 activation is independent of NLRP3 and ASC

The mechanism of caspase- 1 activation is well studied and has been shown to involve NLRP3 and ASC recruitment into a complex. To determine if the NLRP3 inflammasome complex was required for caspase-4 activation, NLRP3 CRISPR/Cas9 KO THP-1 macrophages and WT controls were treated with Eh for 10-60 min and caspase- 4 activation was quantified by densitometric analysis. Caspase-4 activation in NLRP3 KO cells was unaffected (Figs 3a, b). IL-1 $\beta$ protein levels were absent in the NLRP3 CRISPR/Cas9 KO compared to WT THP-1 macrophages, supporting the notion that NLRP3 is the central inflammasome activated by Eh. Caspase-4 levels were similar in THP-1 deficient in ASC (defASC) macrophages when treated with Eh for 10-60 min (Figs 3c, d). THP-1 

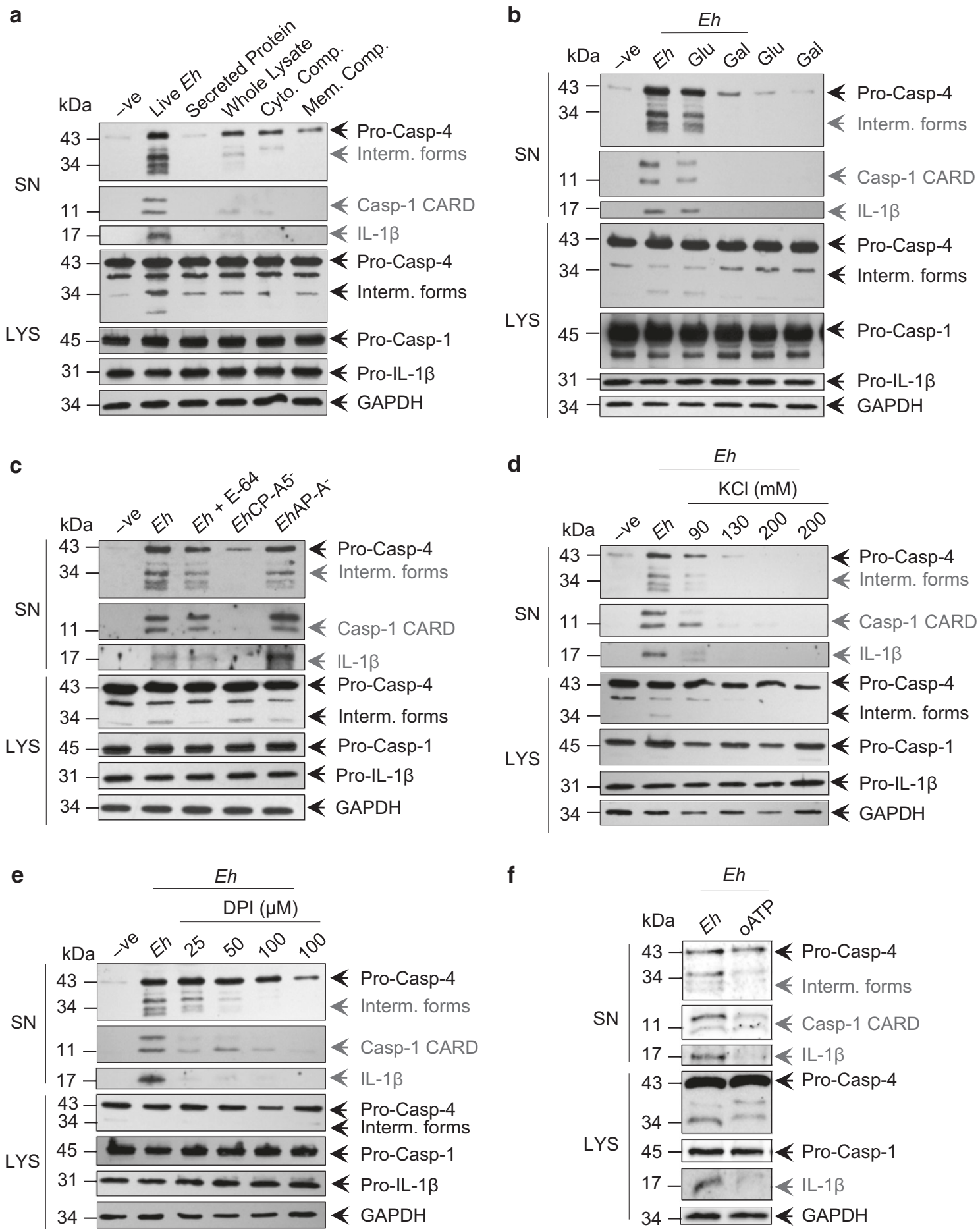

f

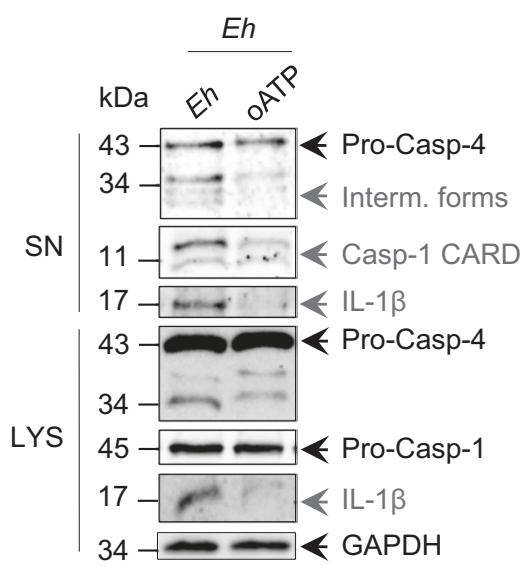

Fig. 2 E. histolytica-induced caspase-4 activation parallels caspase-1 requiring live parasite and contact via Gal-lectin and EhCP-A5 and involves cellular perturbations. a THP-1 macrophages were incubated with different preparations of Eh including live Eh, whole lysates, or with membrane (mem. comp.) or cytoplasmic fractions (cyto. comp.) of equal amounts of Eh for 60 min. b THP- 1 macrophages were pretreated for $5 \mathrm{~min}$ with $55 \mathrm{mM}$ D-galactose (Gal), or glucose (Glu) as an osmotic control and then incubated with Eh for 60 min at a 1:20 ratio (optimal dosage from Fig. 1a). c THP-1 macrophages were incubated with Eh, E-64 treated Eh, and different trophozoites, Eh deficient in CP5 (EhCP-A5 ${ }^{-}$) and Eh deficient in amoebapore as the vector control (EhAP-A ${ }^{-}$) at a 1:20 for 60 min. THP-1 macrophages were pretreated with exogenous d potassium chloride $(\mathrm{KCl})$ or e diphenyleneiodonium chloride (DPI) for $1 \mathrm{~h}$ and then incubated with Eh for 60 min. $\mathbf{f}$ THP-1 macrophages were pretreated with $300 \mu \mathrm{M}$ oxidized ATP (OATP) for $2 \mathrm{~h}$ and then incubated with Eh for $30 \mathrm{~min}$. Cell supernatant was TCA precipitated and cells were washed and lysed. Equal amounts of lysates were loaded onto SDS-PAGE gel and immunoblot analysis was performed for caspase-4, caspase-1, IL-1 $\beta$ in both the supernatants (SN) and lysates (LYS). Blots were reprobed for GAPDH. Western blots are representative of three independent experiments 
a

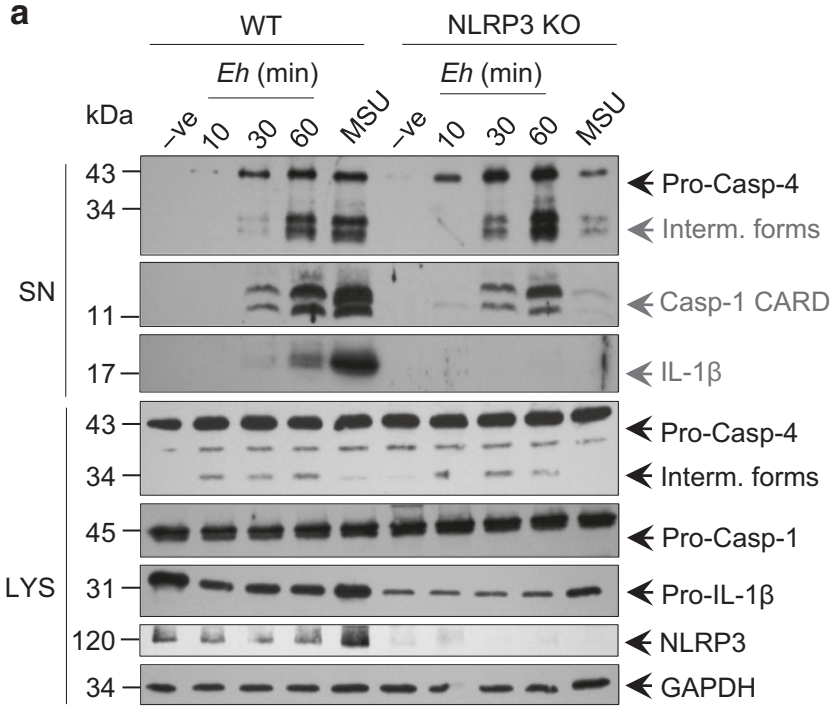

C
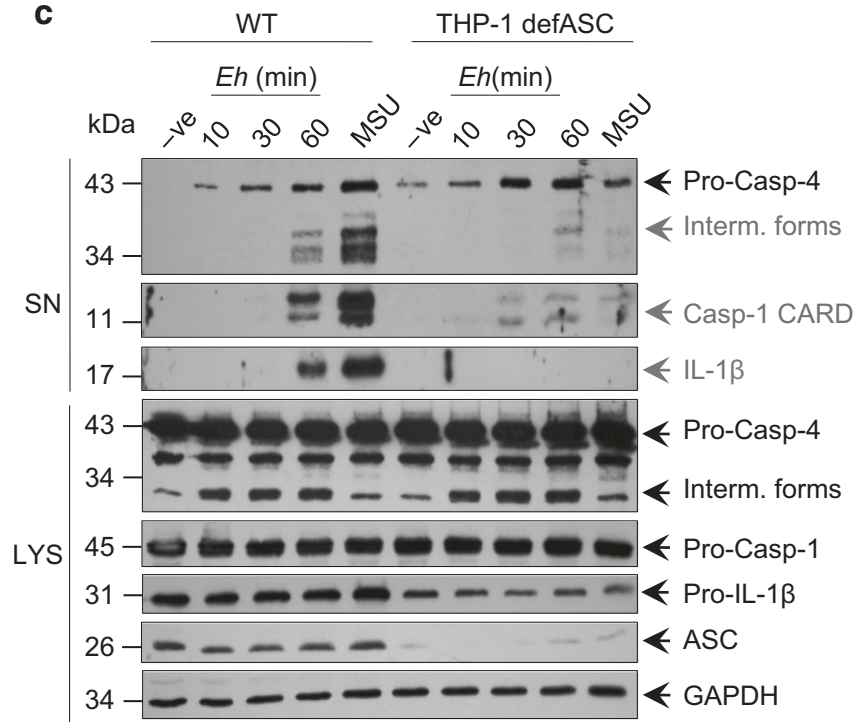

b

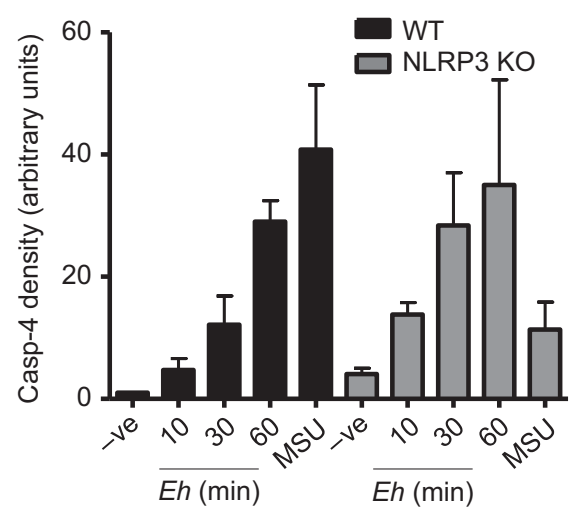

d

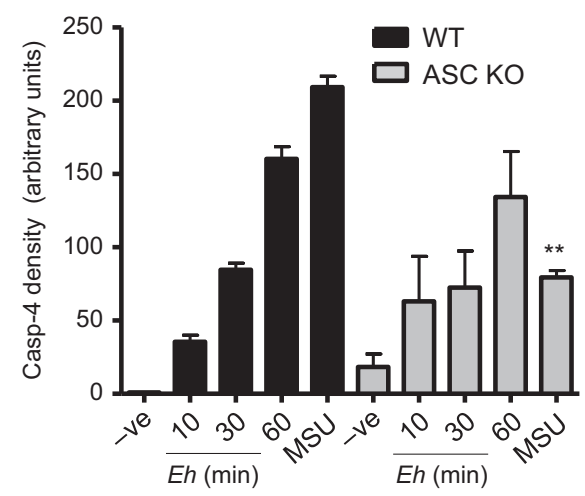

Fig. 3 E. histolytica-induced caspase-4 activation is independent of inflammasome components. a, b WT, NLRP3 CRISPR/Cas9 KO THP-1, and c, d THP-1 defASC macrophages were incubated with Eh (1:20) at increasing time points. Cell supernatant was TCA precipitated and cells were washed and lysed. Equal amounts of lysates were loaded onto SDS-PAGE gel and immunoblotted and immunoblot analysis was performed for caspase-4, caspase-1, and IL-1 $\beta$ in the supernatants (SN) and along with NLRP3 and ASC in lysates (LYS). Blots were reprobed for GAPDH. Quantifications of caspase-4 proteins were performed by densitometric analysis $(\mathbf{b}, \mathbf{d})$ from three independent experiments, and the negative (cells only) acted as an internal control. Data are representative of three experiments and statistical significance was calculated with Student's $t$-test between KO and WT $\left({ }^{* *} p<0.01\right)$. Bars represent \pm SEM

defASC macrophages displayed less caspase-1 CARD and IL-1 $\beta$ secretion, supporting the requirement of the ASC adapter to be recruited to a molecular complex, along with NLRP3 for activating caspase- 1 . In the contrary, MSU was shown to require ASC for caspase- 4 and -1 activation.

As murine caspase-11 is believed to be the ortholog to human caspase-4, we used mouse bone marrow-derived macrophages (BMDM) deficient in Asc and Nlrp3 to explore whether the requirements are similar. Control, $\mathrm{Asc}^{-1-}$ and $\mathrm{Nlrp3}^{-1-}$ BMDM were stimulated with $E h$ for 5, 30, or $60 \mathrm{~min}$. LPS and cholera toxin $B$ (CTB) activates the noncanonical inflammasome and LPS and nigericin (LN) activates the NLRP3 inflammasome, so they were used as positive controls. Robust $E h$-induced caspase- 11 activation was detected in the supernatants at $60 \mathrm{~min}$; however, there was no difference in caspase-11 protein levels (p43 and p38) between $\mathrm{Asc}^{-/-}$and $\mathrm{Asc}^{+/+}$BMDM (Supplementary Figure S3A).
Predictably, cleavage of the caspase- 1 p20 and IL-1 $\beta$ (17 kDa) was absent in $\mathrm{Asc}^{-/-}$showing a dependency of caspase-1 activation on inflammasome complex formation. Additionally, there was also no difference in caspase- 11 protein levels between $\mathrm{Nlrp}^{-/-}$compared to NIrp3 ${ }^{+/+}$BMDM in response to Eh (Supplementary Figure S3B). The cleavage of caspase-1 p20 was absent in NIrp3 $3^{-/-}$and IL-1 $\beta$ was reduced compared to Nlrp3 $3^{+/+}$ BMDM. This indicates that both human caspase- 4 and murine caspase-11 are activated independently of NLRP3 and ASC.

E. histolytica-induced caspase-1 activation is dependent on caspase4 and caspase- 4 activation is independent of caspase- 1

To interrogate whether caspase- 1 required caspase- 4 for its activation, CASP4 CRISPR/Cas9 KO THP-1 macrophages were stimulated with Eh for 10-60 min and monitored for caspase-4 and -1 activation and IL-1 $\beta$ release. Unexpectedly, caspase- 1 


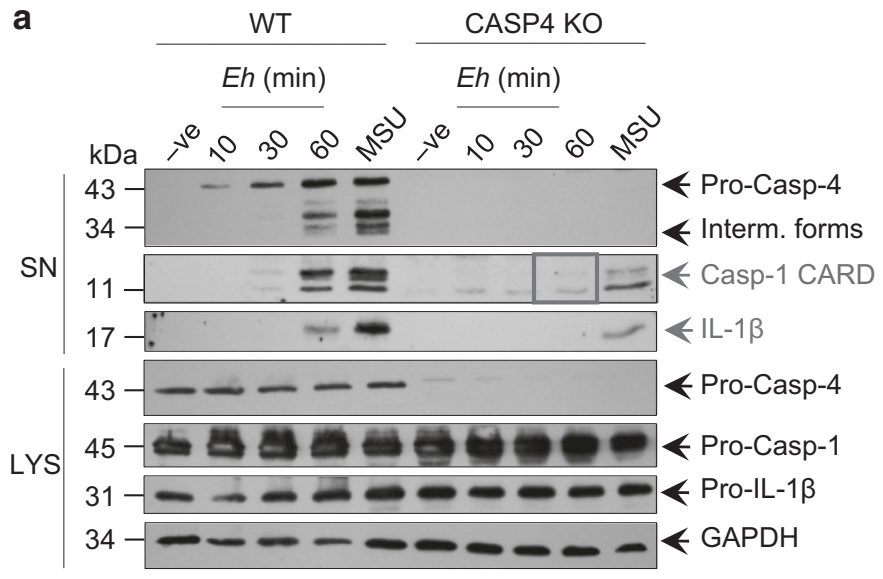

b

C

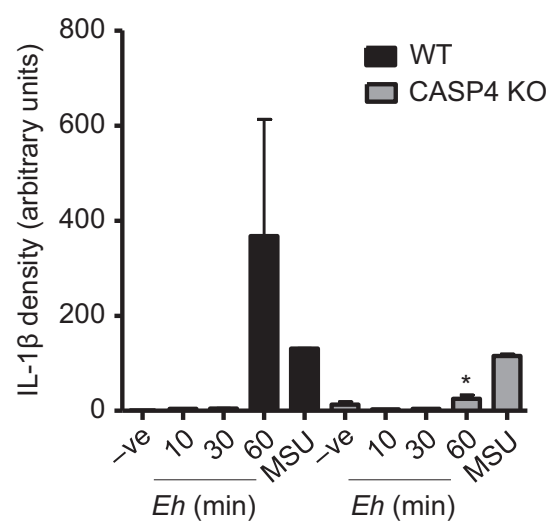

d

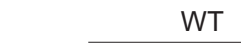

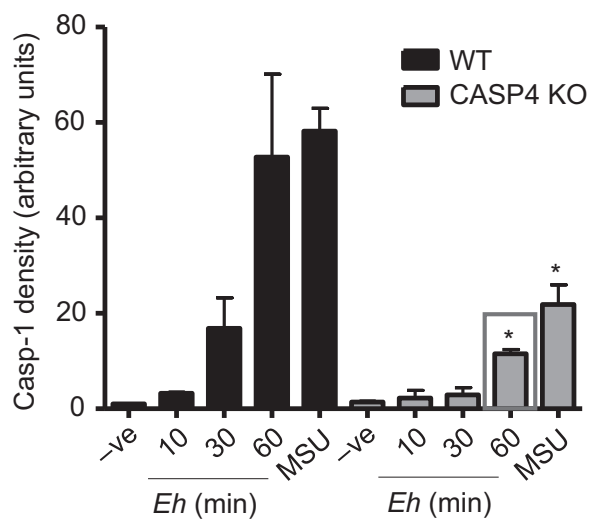
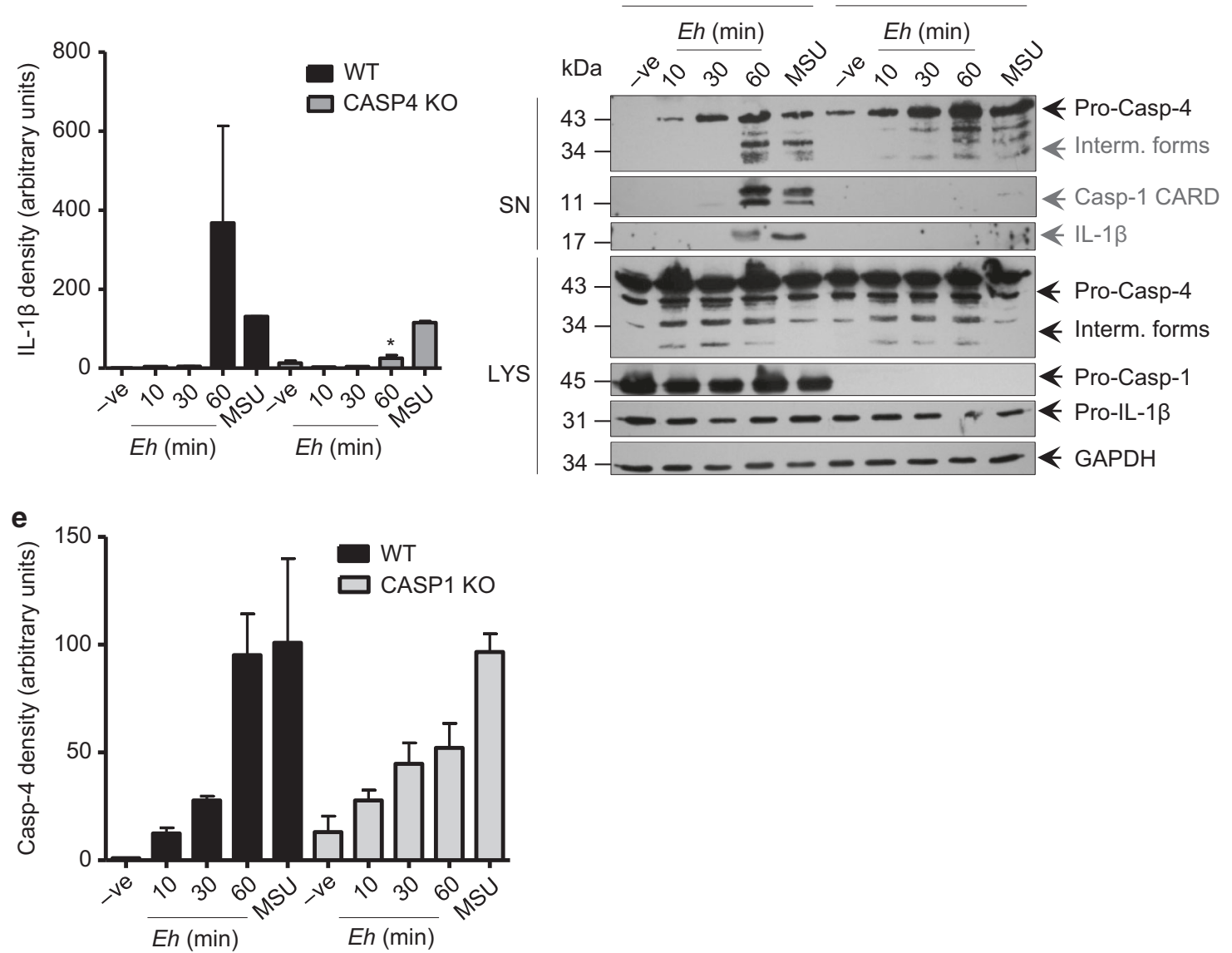

Fig. 4 E. histolytica-induced caspase-1 activation is dependent on caspase-4 and caspase-4 activation is independent of caspase-1. a-c WT, CASP4 CRISPR/Cas9 KO and d, e CASP1 CRISPR/Cas9 KO THP-1 macrophages were incubated with Eh (1:20) at increasing time points. Cell supernatant was TCA precipitated and cells were washed and lysed. Equal amounts of lysates were loaded onto SDS-PAGE gel and immunoblot analysis was performed for caspase-4, caspase-1, IL-1 $\beta$ in both the supernatants (SN) and lysates (LYS). Blots were reprobed for GAPDH. Quantifications of caspase- 1 and IL-1 $\beta$ (b and $\mathbf{c}$ ) and caspase-4 (e) were performed by densitometric analysis from three independent experiments, and the negative (cells only) acted as an internal control. Data are representative of three experiments and statistical significance was calculated with Student's $t$-test between KO and WT $\left({ }^{*} p<0.05\right)$. Bars represent \pm SEM

activation and IL-1 $\beta$ release were significantly less ( $73 \%$ and $97 \%$, respectively, $p<0.05)$ in CASP4 CRISPR/Cas9 KO compared to WT THP-1 macrophages as shown by western blot and densitometric analysis (Figs $4 a-c$; red highlighted box) suggesting a requirement of caspase- 4 in caspase- 1 activation. This requirement of caspase- 4 by caspase-1 was also observed with the positive control MSU (Fig. 4a). A regulatory role of caspase- 4 in caspase- 1 activation was previously shown in keratinocytes transfected with LPS and UVB irradiated $^{21,25,26}$ but no studies to date have shown this response toward a parasitic infection. Furthermore, the orthologue of caspase- 4 found in mouse known as caspase-11, also points to the requirement of caspase-11 in caspase-1-mediated IL-1 $\beta$ secretion in response to Gram-negative bacteria. ${ }^{27}$ To determine whether caspase- 4 activation required caspase-1, CASP1 CRISPR/Cas9 KO 
THP-1 macrophages were stimulated with Eh for 10-60 min. Although there was less IL-1 $\beta$ secretion, there were no significant differences in caspase- 4 activation suggesting that caspase- 1 is not upstream of caspase-4 (Figs $4 d$, e). MSU stimulation also showed less caspase- 1 activation and IL-1 $\beta$ secretion, but no difference in caspase- 4 activation. Taken together, these results support the notion that caspase- 4 is upstream of caspase- 1 to activate IL-1 $\beta$ secretions in response to $E h$. This is in contrast to studies that found reduced secretion of caspase- 4 in caspase- $1 \mathrm{KO}$ in UVB-irradiated keratinocytes, indicating the effects are agonistdependent. ${ }^{21}$ Even though there is high sequence homology among the inflammatory caspases, caspase- 4 and -1 CRISPR/Cas9 KO macrophages did not show off target effects to caspase-1 or -4 , respectively. To confirm a role for caspase- 4 enzymatic activity in caspase- 1 activation, we used Z-LEVD-FMK to inhibit caspase-4 activity in WT macrophages stimulated with $E h$ and it inhibited caspase-1 activation. To test specificity of this inhibitor, we pretreated CASP4 CRISPR/Cas9 KO cells with Z-LEVD-FMK prior to stimulation with $E h$ and it also inhibited caspase- 1 activation. This indicates that the inhibitor is non-specific and that the caspase substrates overlap. ${ }^{28}$

Studies were also done on BMDMs derived from Casp $11^{-1-}$ mice stimulated with $E h$ for 5, 30, and $60 \mathrm{~min}$ and there were no differences in caspase- 1 p20 and IL-1 $\beta$ protein expression as compared to Casp $11^{+/+}$controls (Supplementary Figure S4A). The positive control LPS and CTB showed slightly less caspase-1 activation in Casp $11^{-/-}$as compared to Casp $11^{+/+}$macrophages. These findings indicate that although caspase- 11 has similar parasite requirements in the activation of caspase- 4 , it may serve different functions. Predictably, using $\operatorname{Casp}^{-/-} \operatorname{Casp} 11^{-/-}$there was less IL- $1 \beta$ as compared to Casp $1^{+/+}$Casp $11^{+/+}$macrophages when stimulated with Eh (Supplementary Figure S4B). Caspase-1 activation was not dependent on caspase-11, suggesting that caspase- 4 and caspase- 11 may play different roles in response to Eh.

\section{E. histolytica-induced IL-1 $\beta$ is dependent on caspase- 1 and} caspase-4

To determine the distinct role of caspase- 4 in regulating IL-1 $\beta$ and other pro-inflammatory molecules, a human cytokine array focused 13-plex was used to screen for differences in cytokines/ chemokines in CASP4 CRISPR/Cas9 KO compared to WT macrophages stimulated with $E h$. The most interesting finding was that IL-1 $\beta$ levels were $90 \%$ lower in CASP4 CRISPR/Cas9 KO macrophages, which was consistent with the results seen by western blots of CASP1 and CASP4 CRISPR/Cas9 KO THP-1 macrophages (Figs $4 a, d, 5 a)$. To determine if the $\mathrm{IL}-1 \beta$ released was bioactive, we used the SEAP assay that showed significantly $(p<0.001)$ less bioactive IL-1 $\beta$ released by CASP4 CRISPR/Cas9 KO as compared to WT THP-1 macrophages (Fig. 5b; highlighted histogram), suggesting there is cross talk between caspase- 4 and -1 in regulating IL-1 $\beta$ release. The SEAP assay is likely more sensitive to detect IL$1 \beta$, which explains the presence of $600 \mathrm{pg} / \mathrm{mL}$ of bioactive IL-1 $\beta$, whereas it was not detected by immunoblot. LDH levels in CASP1 and CASP4 CRISPR/Cas9 KO compared to WT THP-1 macrophages were not significantly different when stimulated with Eh from 1 to $3 \mathrm{~h}$, indicating that these caspases do not drive macrophage cell death. The positive control, LPS and nigericin showed differences in cell death in CASP1 and CASP4 CRISPR/Cas9 KO macrophages as compared to WT indicating that both caspase- 1 and -4 activated cell death (Fig. 5c). In CASP4 CRISPR/Cas9 KO macrophages stimulated with LPS and nigericin, there was no difference in caspase-1 CARD cleavage or IL- $1 \beta$ secretion (Figs $5 \mathrm{~d}$, e). This indicates that there is no requirement for caspase-4 in activating caspase-1. Prior to obtaining CRISPR/Cas9 KO, siRNA silencing of caspase- 4 showed no differences in caspase- 1 activation in response to $E h$ even though there were reduced IL-1 $\beta$ levels in caspase- 4 siRNA compared to scrambled siRNA
(Supplementary Figures S2A and S2B). Due to incomplete knockdown, minimal caspase-4 protease activity was still sufficient to activate caspase-1, hence there were no differences observed with caspase- 1 protein levels. The siRNA experiments did support the results obtained from the CASP4 CRISPR/Cas9 KO macrophages, that IL-1 $\beta$ levels were reduced in caspase-4-deficient macrophages stimulated with Eh compared to control cells.

Overexpression of caspase- 4 rescued $E$. histolytica-induced IL-1 $\beta$ secretion in CASP4 CRISPR/Cas9 KO macrophages

To confirm that caspase-4 had a role in enhancing IL-1 $\beta$ secretion, CASP4 CRISPR/Cas9 KO THP-1 macrophages were nucleofected with pro-caspase-4 expression plasmid and stimulated with $E h$ for $60 \mathrm{~min}$. As indicated on the western blot, there was efficient restoration of pro-caspase- 4 band ( 41 $\mathrm{kDa}$ ) slightly lower than the endogenous form of pro-caspase-4 in WT macrophages (Fig. 6a). This $41 \mathrm{kDa}$ pro-caspase-4 is processed by Eh into a $36 \mathrm{kDa}$ protein that is observed to align with the protein in WT macrophages. The protein band at 45 $\mathrm{kDa}$ in the CASP4 CRISPR/Cas9 KO macrophage could be a nonfunctional protein recognized by the antibody. There was also higher caspase- 1 and IL-1 $\beta$ protein expression in the CASP4 CRISPR/Cas9 KO macrophages treated with Eh compared to non-stimulated control cells. Strikingly, there was increased caspase- 1 activation (highlighted box) and IL-1 $\beta$ protein expression in CASP4 CRISPR/Cas9 KO transfected with the caspase-4 plasmid when stimulated with $E h$ as compared to non-stimulated transfected CASP4 CRISPR/Cas9 KO THP-1 macrophages. More importantly, bioactive IL-1 $\beta$ secretion was rescued when pro-caspase-4 was overexpressed in CASP4 CRISPR/Cas9 KO THP-1 macrophages and stimulated with Eh as compared to control ( $p<0.01$, Fig. $6 \mathrm{~b}$; highlighted histogram). These results suggest that overexpressed pro-caspase-4 when stimulated with Eh stimulation enhanced caspase-1 processing at the CARD domain to enhance caspase-1dependent maturation of IL-1 $\beta$. This is likely mediated by the proteolytic activity of caspase-4, as this is needed in order to activate caspase- 1 and subsequently IL-1 $\beta$ secretions. WT macrophages transfected with pro-caspase-4 plasmid also upregulated IL-1 $\beta$ secretion in response to $E h$ and ATP (Supplementary Figure S5A). When CASP1 CRISPR/Cas9 KO THP-1 macrophages were transfected with the caspase-1 plasmid, there was no caspase-1 CARD cleavage (Supplementary Figure S5B) or bioactive IL-1 $\beta$ secretion (Supplementary Figures S5B and S5C) because the majority of transfected cells had died.

E. histolytica-induced GSDMD cleavage regulates IL-1 $\beta$ secretion To determine if $E$-induced caspase- 4 and -1 activation cleaved GSDMD, THP-1 macrophages were stimulated with Eh from 10 to $180 \mathrm{~min}$. As early as $10 \mathrm{~min}$ Eh stimulation, the $55 \mathrm{kDa}$ GSDMD was cleaved into the $\mathrm{N}$-terminal p30 fragment present in cell lysates (Fig. 7a). The appearance of GSDMD p30 in the supernatant only occurred after $60 \mathrm{~min}$ of Eh stimulation indicating low levels of cell death. In comparison, the potent agonist for GSDMD cleavage, LPS and nigericin (LN) after $60 \mathrm{~min}$ resulted in the accumulation of GSDMD in both the cell lysate and supernatant (Fig. 7a). To determine if there was a dependency for GSDMD cleavage by caspase- 4 or -1, CASP1 and CASP4 CRISPR/Cas9 KO macrophages were stimulated with $E h$ for $30 \mathrm{~min}$. There was no difference between CASP1 KO and WT THP-1 macrophages in GSDMD cleavage; however, there was reduced GSDMD cleavage in CASP4 KO compared to WT THP-1 macrophages in response to Eh (Fig. 7b). This suggests that caspase- 4 was more efficient at cleaving GSDMD. In contrast, LPS and nigericin required caspase-1 for GSDMD cleavage. With GSDMD CRISPR/Cas9 KO THP-1 macrophages, there was less caspase- 4 secreted in response to $E h$ as compared to WT 

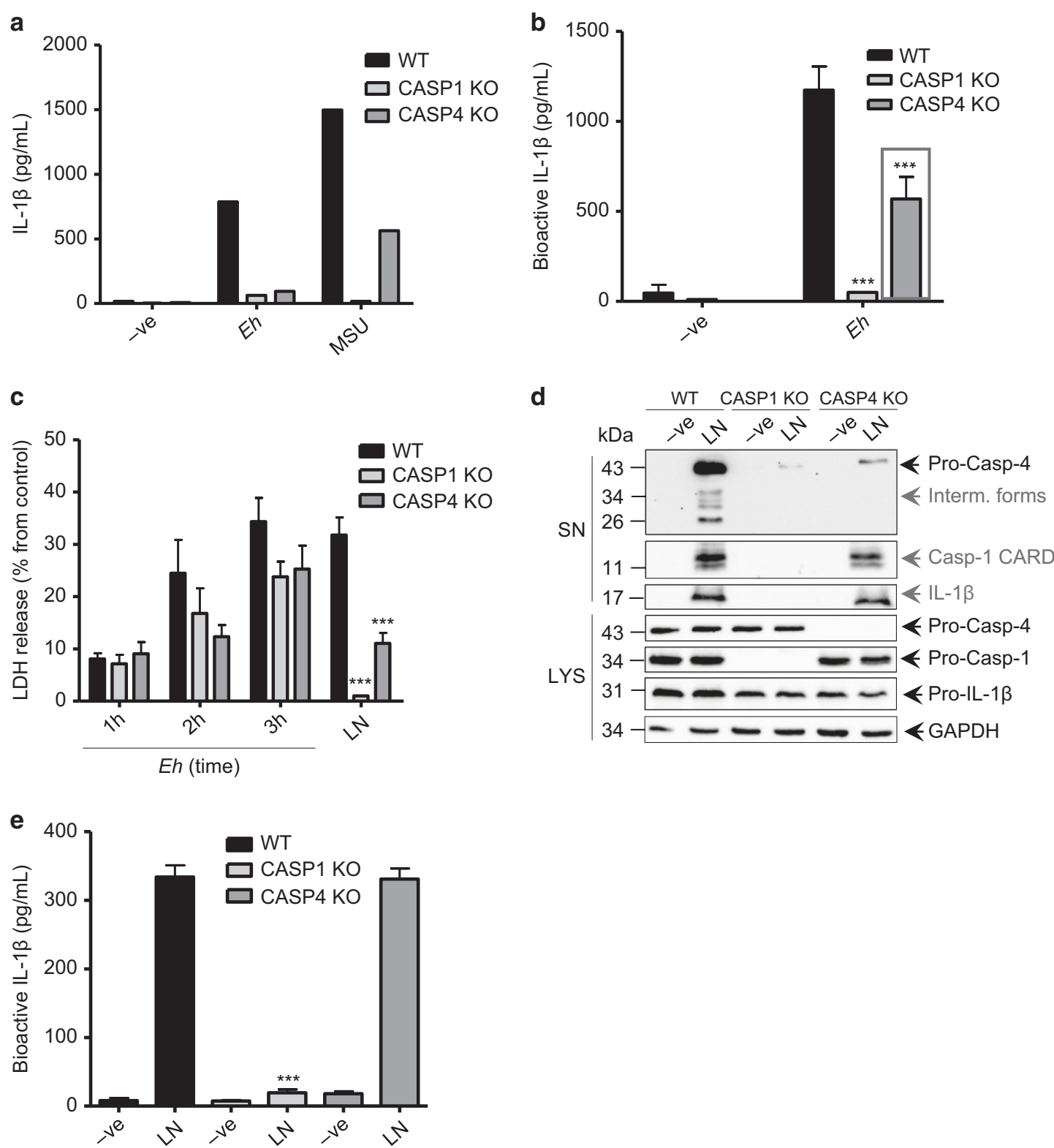

Fig. 5 E. histolytica-induced IL-1 $\beta$ secretion is dependent on caspase- 4 and -1 activation. a WT, CASP4 CRISPR/Cas9 KO and CASP1 CRISPR/Cas9 KO THP- 1 macrophages were incubated with Eh for $60 \mathrm{~min}$ or MSU $(300 \mu \mathrm{g} / \mathrm{mL}$ for $6 \mathrm{~h})$ and cell supernatant was quantified by human focused 13-plex cytokine/chemokine arrays. b Cell supernatant was added to HEK-Blue ${ }^{\mathrm{TM}}$ reporter cells to detect bioactive IL-1 $\beta$ using the SEAP assay. c Cell supernatant was used to measure cell death by LDH release and is shown as a percentage of LDH release from cells without stimulation (control). LN is abbreviated for LPS $(50 \mathrm{ng} / \mathrm{mL})$ and nigericin $(10 \mu \mathrm{M})$ stimulation for $30 \mathrm{~min}$. d WT, CASP4 CRISPR/Cas9 KO and CASP1 CRISPR/ Cas9 KO THP-1 macrophages were incubated with LPS $(50 \mathrm{ng} / \mathrm{mL})$ and nigericin $(10 \mu \mathrm{M})$ stimulation for 60 min. Equal amounts of lysates were loaded onto SDS-PAGE gel and immunoblot analysis was performed for caspase-4, caspase-1, IL-1 $\beta$ in both the supernatants (SN) and lysates (LYS). Blots were reprobed for GAPDH. e Cell supernatant was added to HEK-Blue ${ }^{\mathrm{TM}}$ reporter cells to detect bioactive IL-1 $\beta$ using the SEAP assay. Data are representative of three experiments and statistical significance was calculated with ANOVA and Bonferroni's post-hoc test $(* * * 00.001)$. Bars represent \pm SEM

macrophages, but no difference in caspase-1 activation (Fig. 7c). GSDMD could be involved in caspase- 4 secretion, as the mechanism of its secretion is unknown. Bioactive Eh-induced IL-1 $\beta$ secretion was $80 \%$ less $(p<0.001)$ in GSDMD KO as compared to WT macrophages (Figs $7 c, d)$. This indicates that GSDMD is downstream of caspase-4 and -1 , but upstream of IL-1 1 . LDH release was also measured to determine cell death in GSDMD KO macrophages stimulated with $E h$, and there were significantly higher levels of LDH release, indicating that IL-1 $\beta$ release was not due to cell death or caspasemediated cell death termed pyroptosis (Fig. 7e). GSDMD KO macrophages treated with LPS and nigericin showed that IL-1 $\beta$ release was dependent on GSDMD and that these cells are resistant to pyroptosis. To validate the specificity for GSDMD in mediating IL$1 \beta$ release, a peptide inhibitor, $N$-acetyl-Phe-Leu-Thr-Asp-chloromethylketone (Ac-FLTD-CMK) ${ }^{29}$ was used to inhibit GSDMD cleavage by caspases- $1,-4$, and -5 . Pretreatment of macrophages with the inhibitor blocked Eh-induced IL-1 $\beta$ secretion at 10 and $20 \mu \mathrm{M}$ (Supplementary Figure S5D and S5E). Both inhibitor and genesilenced cells indicate the importance of GSDMD in mediating IL-1 $\beta$ secretion in response to $E h$. 

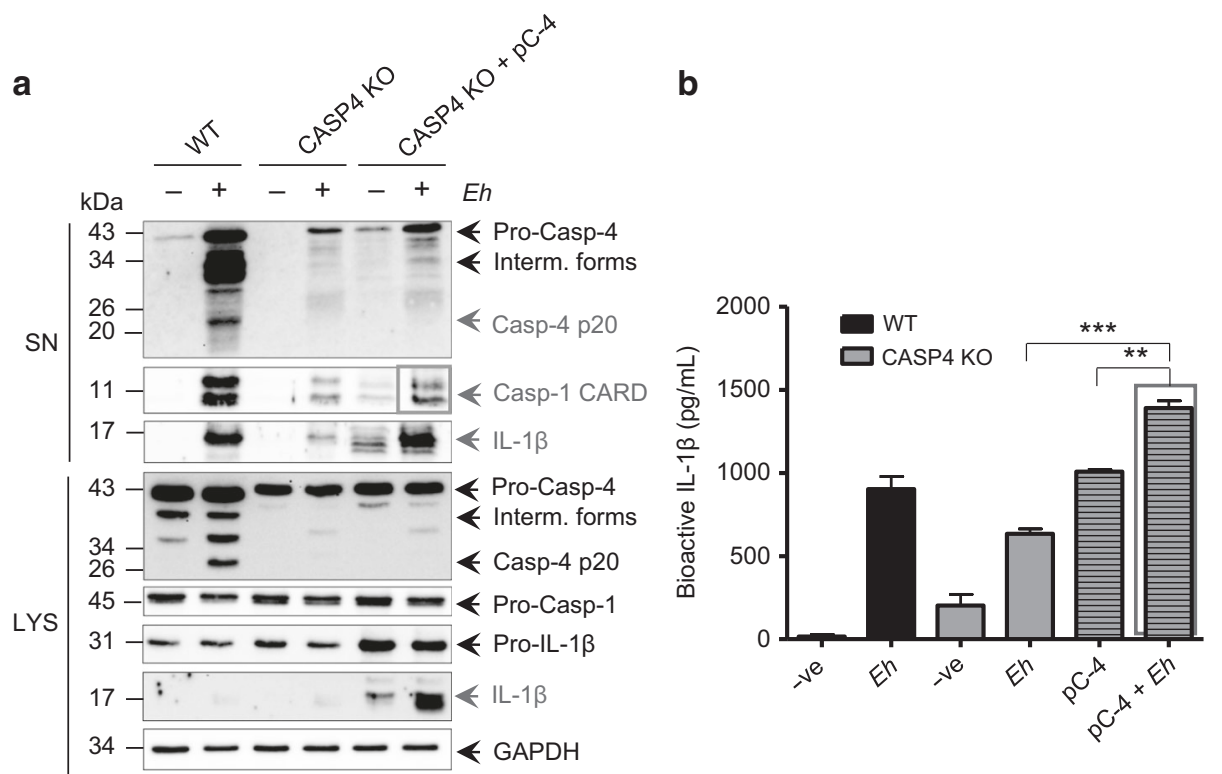

Fig. 6 Overexpression of caspase-4 rescues $E$. histolytica-induced IL-1 $\beta$ secretion in CASP4-deficient macrophages. a CASP4 CRISPR/Cas9 KO THP-1 macrophages were nucleofected with caspase-4 expression plasmid (abbreviated pC-4) and left to rest for $24 \mathrm{~h}$ before incubating with Eh (1:20) for 60 min. b Cell supernatant from stimulated macrophages was also added to HEK-Blue ${ }^{\mathrm{TM}}$ reporter cells to detect bioactive IL-1 $\beta$ using the SEAP assay. Cell supernatant was TCA precipitated and cells were washed and lysed. Equal amounts of protein were loaded onto SDS-PAGE gel and immunoblot analysis was performed for caspase-4, caspase-1, and IL-1 $\beta$ in both the supernatants (SN) and lysates (LYS), and blots were reprobed for GAPDH. Data are representative of three experiments and statistical significance was calculated with Student's $t$-test $\left({ }^{* *} p<0.01,{ }^{* * *} p<0.001\right)$. Bars represent \pm SEM

E. histolytica-induced IL-1 $\beta$ secretion is dependent on caspase-4 interaction with caspase-1

To determine if the caspases specifically interacted with each other, COS-7 cells were transfected with pro-caspase-1, procaspase- 4 , and pro-IL-1 $\beta$ plasmids. We used COS-7 cells as they are easy to transfect with more than $90 \%$ transfection efficiency using GFP and they do not express ASC or NLRP3, facilitating the interactions between the caspases independent of the inflammasome complex (Fig. 8a). Although weak pro-caspase-1 and procaspase- 4 were detected by western blot under basal conditions, they were not activated unless stimulated with Eh. Transfection with GFP served as the control. When pro-caspase-1, pro-caspase4 , and pro-IL-1 $\beta$ plasmids were transfected and stimulated with Eh, pro-caspase- 1 was cleaved into active caspase-1 p20 fragment and pro-caspase- 4 was cleaved into the large subunit, both of which were detectable in cell lysates $(26 \mathrm{kDa}$, highlighted box; Fig. 8a). This subsequently led to the cleavage of IL-1 $\beta$ from 31 to $17 \mathrm{kDa}$. LPS and ATP stimulation (abbreviated LA) did not yield detectable caspase- 4 or -1 activation in the supernatant, but was present in the lysates (Fig. 8b). This indicates a similar intracellular inflammatory pathway was initiated and LA served as another agonist for caspase-4-caspase- 1 interaction in mediating IL-1 $\beta$ release. Although differences in protein expression were not detectable for the caspases under Eh stimulation, the SEAP assay yielded significant IL-1 $\beta$ differences. Importantly, the transfection of all three plasmids led to an additive effect, with significantly more bioactive IL-1 $\beta$ secretion $(p<0.01)$ than transfection of caspase- 1 and IL-1 $\beta$ plasmids or the transfection of caspase- 4 and IL-1 $\beta$ with Eh stimulation (Fig. 8c; highlighted histogram). As a positive control, LPS was used to prime cells and then stimulated with ATP (LA) in cells transfected with all three plasmids (Fig. 8b). The combination of all three plasmids yielded greater caspase- 1 activation and IL-1 $\beta$ secretion. This provides further evidence that the presence of caspase- 4 enhances caspase- 1 activation and therefore higher amounts of IL-1 $\beta$ secretion. In our study, the use of COS-7 cells was advantageous as they basally express inactive caspase- 4 and -1 (both are pro-forms) while COS- 1 cells $^{21}$ basally expressed active caspase- 4 as indicated by the intermediate forms (Fig. 8d). COS-7 cells allowed us to interrogate the effect of Ehinduced activation of caspases.

Pro-caspase-4 interacts with pro-caspase-1 in Eh-stimulated THP-1 macrophages

To confirm that caspase- 4 was directly interacting with caspase-1, THP-1 macrophages were stimulated with Eh for 20 min followed by $0.8 \%$ formaldehyde treatment for $7 \mathrm{~min}$ at room temperature to covalently cross-link and stabilize the protein-protein interactions. ${ }^{30}$ Antibodies against caspase- 4 and -1 were then used to pull-down (PD) the complex and immunoblotted for caspase-4 and -1 (Fig. 9a). This method is valuable because it allows for the detection of transient interacting proteins. However, as detection of cross-linked proteins is highly dependent on the antibody, we screened several caspase- 4 antibodies from different companies and observed a high molecular weight complex around $92 \mathrm{kDa}$ (cross-linked complex) only with the caspase-4 M029-3 antibody and not with the others (Supplementary Figure S6A). The crosslinked complex was also observed with Coomassie blue staining (Supplementary Figure S6B). The control mouse IgG antibody detected the heavy $(50 \mathrm{kDa})$ and light $(25 \mathrm{kDa})$ chains. We also screened several caspase-1 antibodies and decided to use caspase-1 sc-56036 rather than caspase- 1 sc- 622 because there was less background (Supplementary Figure S6C). The cross-linked complex containing caspase- 4 was detected at $92 \mathrm{kDa}$ when the sample was incubated at $65^{\circ} \mathrm{C}$ and rapidly dissociated when the sample was boiled to $99^{\circ} \mathrm{C}$ demonstrating specificity for the protein-protein complex (Fig. 9b). Pro-caspase-4 was also detected using the caspase-4 M029-3 antibody (Fig. 9b). Immunoprecipitation with caspase-1 sc-56036 antibody following stimulation with $E h$ and formaldehyde treated, detected procaspase-1 (Fig. 9c) and when immunoblotted for caspase-4 detected a band at $43 \mathrm{kDa}$ (Figs 9d, e; highlighted box) indicating that pro-caspase- 1 engages pro-caspase- 4 . 
a

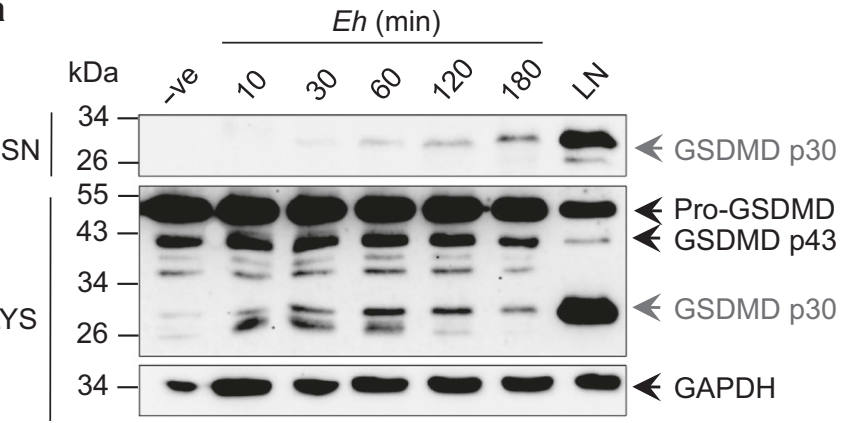

b

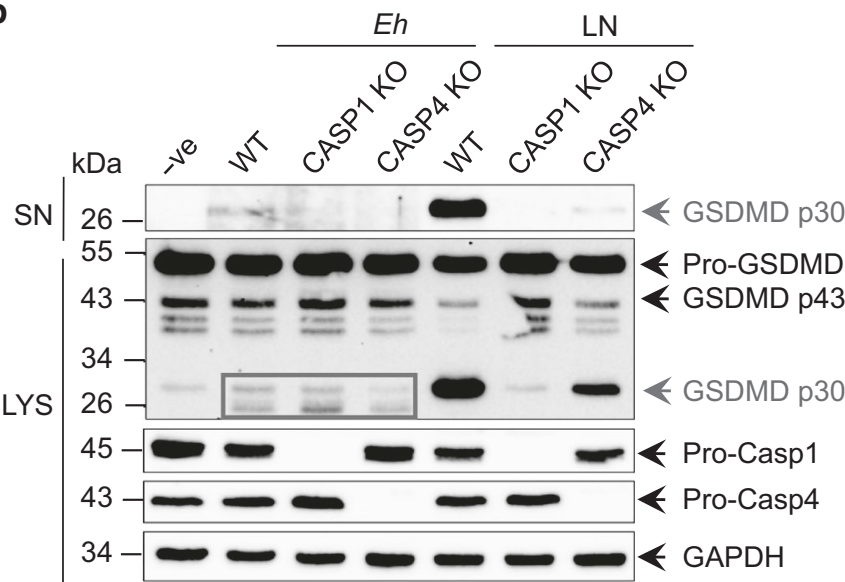

C

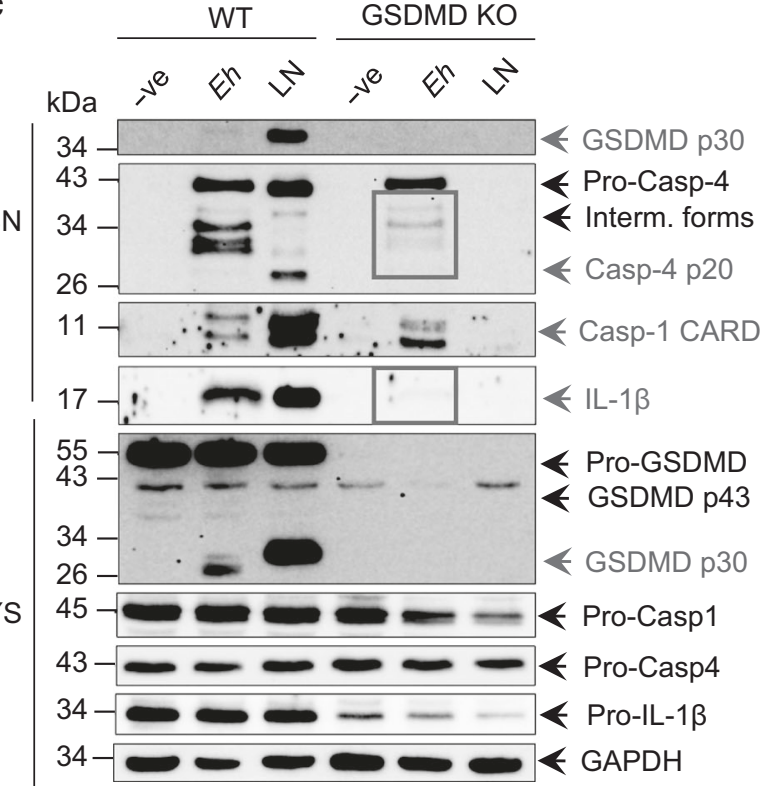

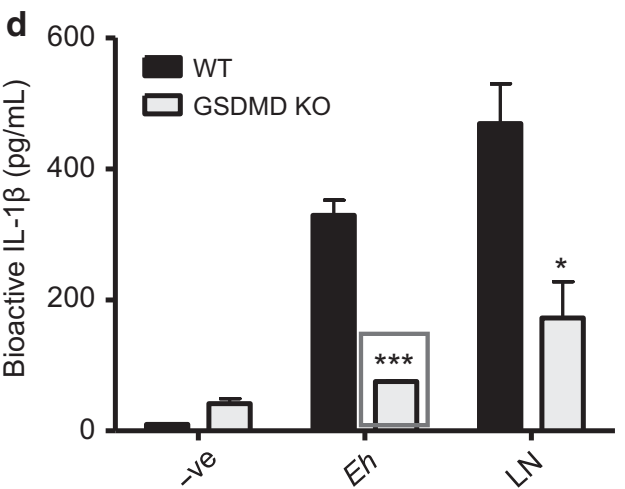

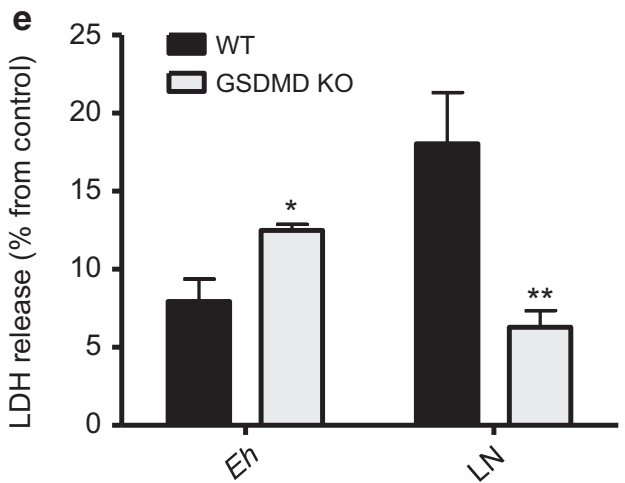

Fig. 7 E. histolytica-induced gasdermin D cleavage regulates IL-1 $\beta$ secretion. a THP-1 macrophages were incubated for increasing amounts of time with 1:20 Eh to macrophage ratio. LPS $(50 \mathrm{ng} / \mathrm{mL})$ and nigericin $(10 \mu \mathrm{M})$ stimulation for 60 min was used as a positive control (abbreviated as LN). b WT, CASP1 CRISPR/Cas9 KO, and CASP4 CRISPR/Cas9 KO macrophages were treated with Eh for 30 min. c WT and GSDMD CRISPR/ Cas9 KO macrophages were incubated with Eh for $30 \mathrm{~min}$. LPS $(50 \mathrm{ng} / \mathrm{mL})$ and nigericin $(10 \mu \mathrm{M})$ stimulation for 30 min was used as the positive control. Cell supernatant from stimulated macrophages was also added to HEK-Blue ${ }^{T M}$ reporter cells to detect bioactive IL-1 $\beta$ using the SEAP assay (d) and percentage of LDH release compared to non-stimulated cells (control) (e). Equal amounts of supernatants and lysates were loaded onto SDS-PAGE gel and immunoblot analysis was performed for GSDMD, caspase-4, caspase-1, and IL-1 $\beta$ in both the supernatants (SN) and lysates (LYS), and blots were reprobed for GAPDH. Data are representative of three experiments and statistical significance was calculated using Student's $t$-test $\left({ }^{*} p<0.05,{ }^{* *} p<0.01,{ }^{* * *} p<0.001\right.$, ns not significant). Bars represent \pm SEM 


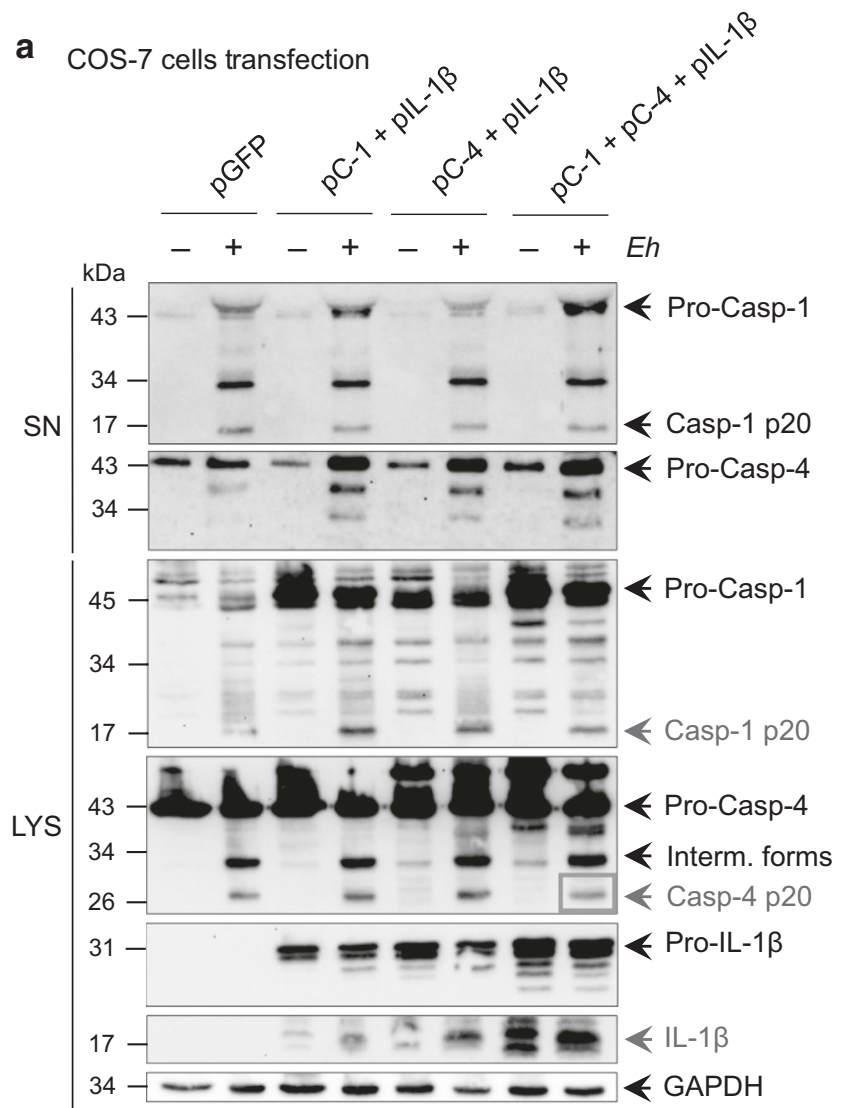

b

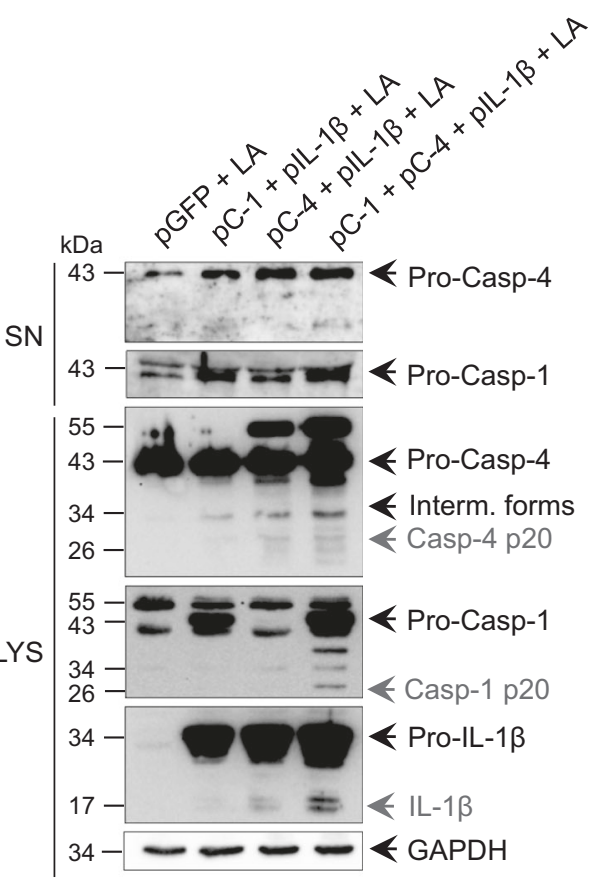

C

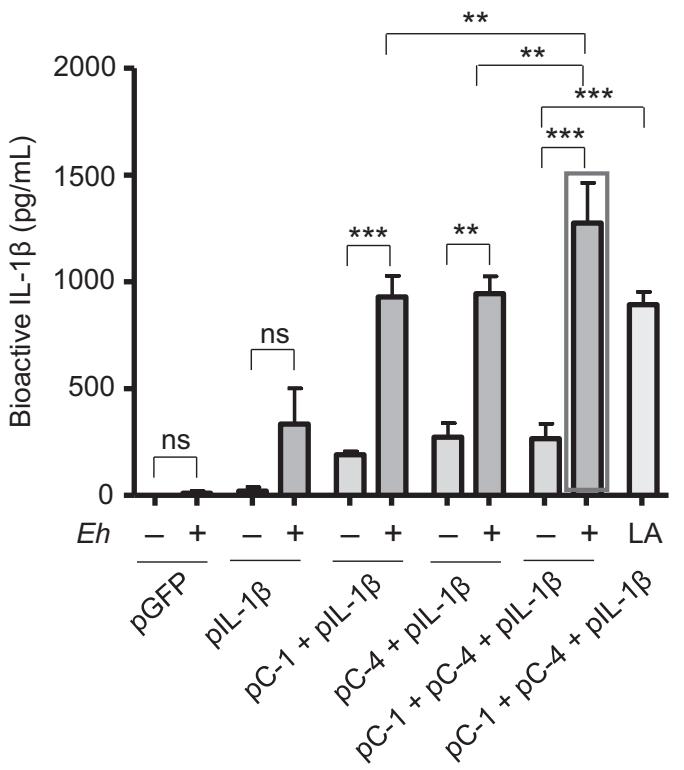

d

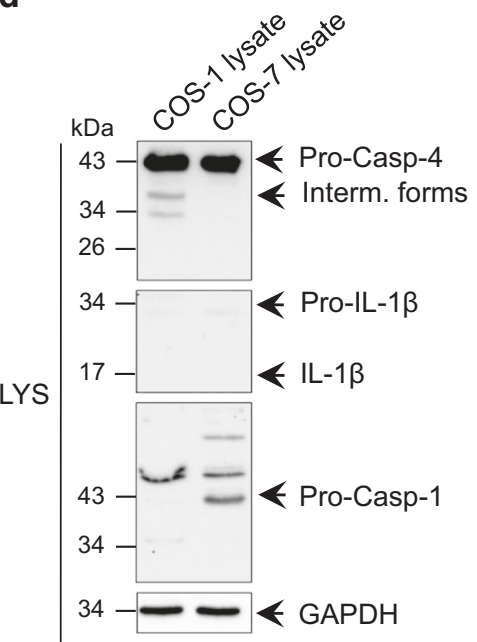

Fig. 8 E. histolytica-induced IL-1 $\beta$ secretion is dependent on caspase-4 interaction with caspase-1. a COS-7 cells were transfected with procaspase-1 (pC-1, $100 \mathrm{ng}$ ), pro-caspase-4 (pC-4, $10 \mathrm{ng})$, pro-IL-1 $\beta$ (plL-1 $\beta, 1 \mu \mathrm{g})$ plasmids, or GFP (pGFP, $1 \mu \mathrm{g})$ as a control and stimulated with Eh (1:2) for $30 \mathrm{~min}$ or b LPS $(50 \mathrm{ng} / \mathrm{mL}$ for $2 \mathrm{~h}$ to prime) and ATP ( $3 \mathrm{mM}$ for $60 \mathrm{~min}$ ) abbreviated LA, served as the control for IL-1 $\beta$ release. c Cell supernatant from stimulated macrophages was added to HEK-Blue ${ }^{T M}$ reporter cells to detect bioactive IL-1 $\beta$ using the SEAP assay. $\mathbf{d}$ Protein expression of caspase-4, caspase-1, IL-1 $\beta$ was compared in COS-1 and COS-7 lysates to indicate basal expression and rationale for usage of COS-7 cells. Cell supernatant was TCA precipitated and cells were washed and lysed. Equal amounts of lysates were loaded onto SDS-PAGE gel and immunoblot analysis was performed for caspase- 4 and -1 in the supernatants (SN) and along with IL-1 $\beta$ in the lysates (LYS). Blots were reprobed for GAPDH. Data are representative of three experiments and statistical significance was calculated with ANOVA and Bonferroni's post-hoc test $\left({ }^{* *} p<0.01,{ }^{* * *} p<0.001\right.$, ns not significant). Bars represent \pm SEM 
J Quach et al.

a

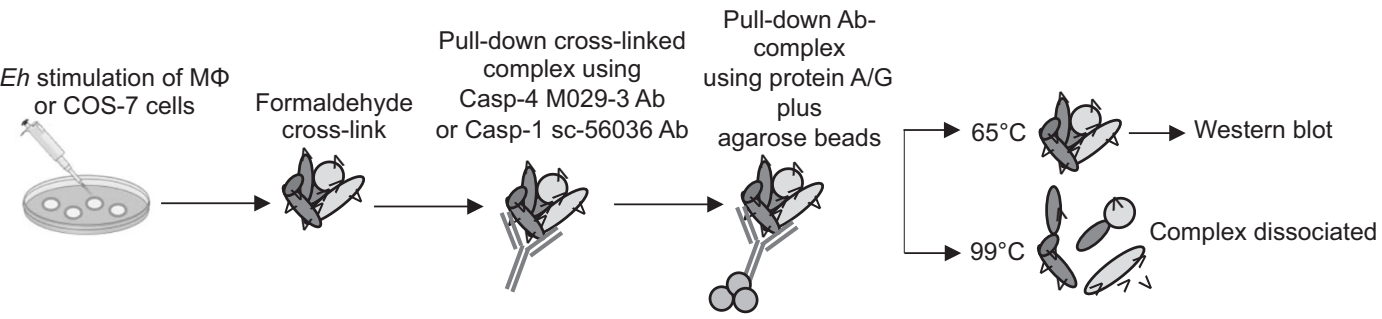

b

THP-1 MФ

PD

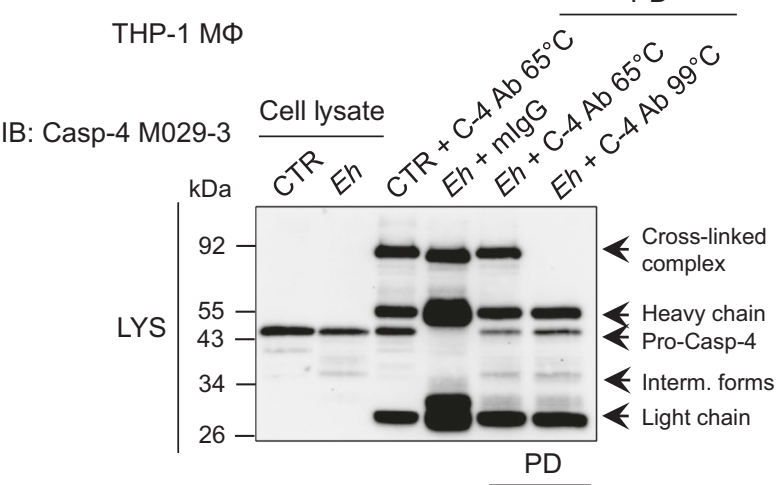

d $\quad$ THP-1 MФ

IB: Casp-4 M029-3
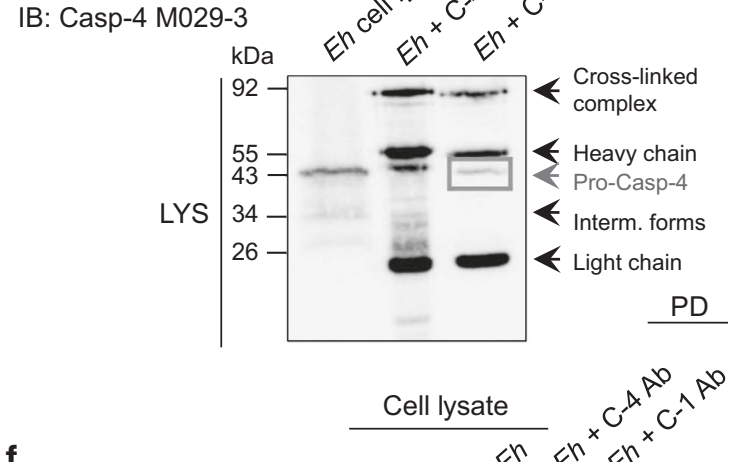

f

Cos-7 cells

IB: Casp-4 M029-3

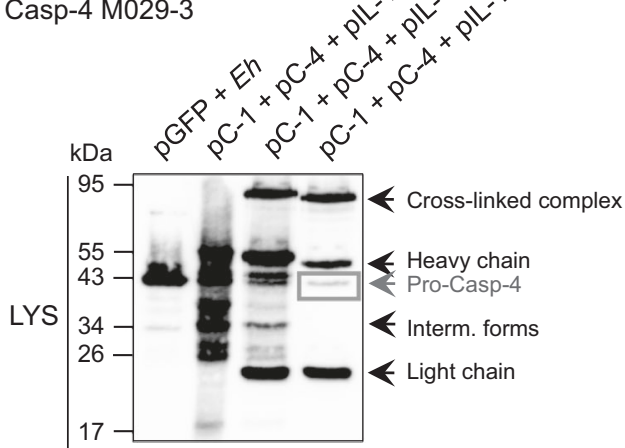

C

THP-1 MФ
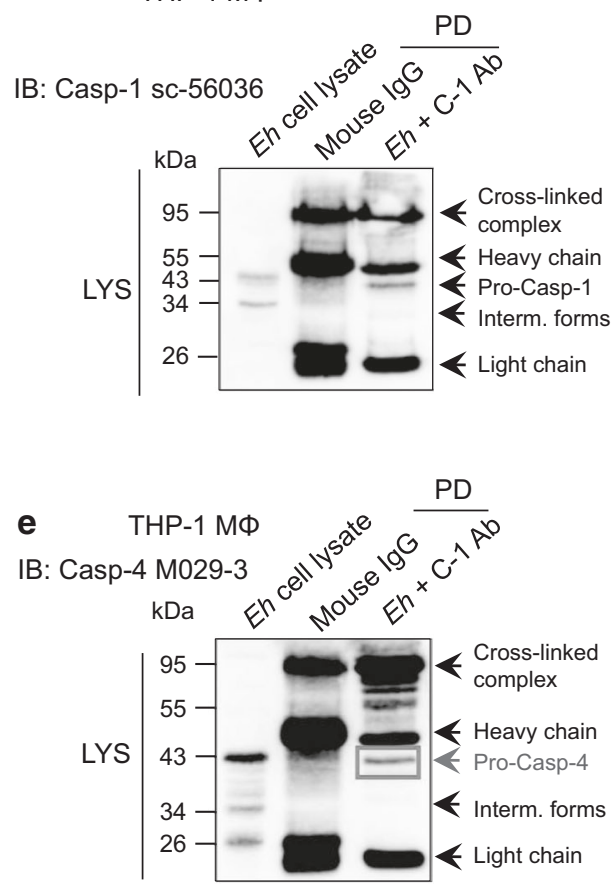

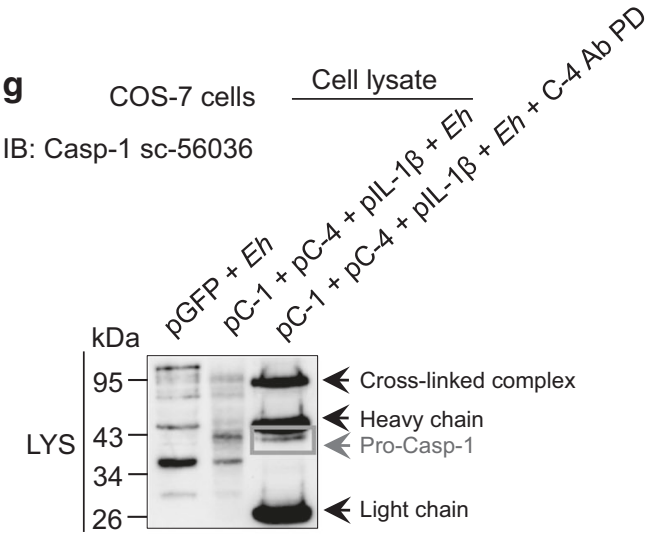

Fig. 9 Pro-caspase-4 interacts with pro-caspase-1 in E. histolytica-stimulated THP-1 macrophages. a A schematic workflow of cell preparations to quantify caspase- 1 and caspase- 4 interaction by pull-down and western blot. Cells were incubated with Eh, treated with formaldehyde, lysed and cross-linked complexes (black triangles with oval shapes) were pulled-down (PD) with antibodies (Y-shaped) and protein A/G plus agarose beads (gray circles). b-e THP-1 macrophages or COS-7 cells were incubated with Eh for 20 min and $0.8 \%$ formaldehyde treated for 7 $\mathrm{min}$ at room temperature. Cells were subjected to pull-down (PD) with caspase-4 M029-3 or caspase-1 sc-56036 antibody at $1.5 \mu \mathrm{g}$ and immunoblotted for caspase-4 or caspase-1. f, g COS-7 cells were transfected with caspase-1 (pC-1, $60 \mathrm{ng}$ ), caspase-4 (pC-4, 500 ng), or IL-1 $\beta$ (pIL-1 $\beta, 1.5 \mu \mathrm{g}$ ) plasmids, or GFP (pGFP, $1 \mu \mathrm{g}$ ) as a control prior to incubating with Eh $(2: 1)$. Cells were subjected to pull-down with caspase-4 M029-3 or caspase- $1 \mathrm{sc}-56036$ antibody at $1.5 \mu \mathrm{g}$ and immunoblotted for caspase- 4 or caspase-1. Corresponding mouse (mlgG) controls were used. Lysates were loaded onto SDS-PAGE gel and immunoblotted with the indicated antibodies. Western blots are representative of three independent experiments 
To validate these observations, COS-7 cells were transfected with pro-caspase- 1 , pro-caspase- 4 , and pro-IL-1 $\beta$ plasmids and stimulated with $E h$ and pulled-down using both caspase-4 and -1 antibodies. As predicted, the high molecular weight cross-linked complex was detected at $92 \mathrm{kDa}$ and when cells were subjected to caspase-1 pull-down, pro-caspase-4 was detected (Fig. 9f; highlighted box). Conversely, when cells were subjected to caspase-4 pull-down, pro-caspase-1 was detected (Fig. 9g; highlighted box). These results provide compelling evidence that caspase- 1 and caspase-4 directly interacted with each other.

\section{DISCUSSION}

This study addressed the distinct roles of inflammatory caspases in Eh-macrophage interaction in the regulation of IL-1 $\beta$. Four different aspects of host caspase-4 and GSDMD activation in Ehmacrophage interaction was studied: (1) was caspase-4 activated in a manner similar to caspase- 1 using the same parasite factors, (2) did caspase- 4 act upstream to regulate caspase- 1 activation and IL-1 $\beta$ secretion or vice versa, (3) was the function of caspase- 4 distinct from caspase-1, and (4) was GSDMD involved in IL-1 $\beta$ secretion. We found that the requirements for activating caspase-4 were the same as caspase- 1 in that live Eh via Gal-lectin contact and EhCP-A5 was necessary for initiating the inflammatory cascade in macrophage leading to high output IL-1 $\beta$. However, the most important finding was that Eh-induced caspase-4 activation specifically interacted with caspase- 1 to enhance the cleavage of the CARD domains amplifying the release of bioactive IL-1 $\beta$ mediated through the cleavage of GSDMD by both inflammatory caspases. This study shows a complex host-parasite

interaction where Eh-induced inflammatory caspases can converge onto one role to regulate high output proinflammatory responses in disease pathogenesis.

Importantly, while intracellular LPS and Gram-negative bacterial pathogens can activate caspase- $4,{ }^{14,31}$ our study shows that an extracellular parasite can induce caspase- 4 activation through outside-in signaling. This suggests that there are several signals that are sensed by caspases. Most notably, for NLRP3 inflammasome activation, these signals include perturbations to ion concentrations such as the drop in cytosolic $\mathrm{K}^{+}$or the production of ROS. Potassium efflux has been shown to be the minimal requirement by many agonizts to activate the NLRP3 inflammasome. ${ }^{32}$ We have recently shown that EhCP-A5 RGD motif engages $a_{5} \beta_{1}$ integrin to induce ATP release through pannexin- 1 channels that signal through $\mathrm{P}_{2} \mathrm{X}_{7}$ receptor to activate the NLRP3 inflammasome. ${ }^{9}$ Both $\mathrm{K}^{+}$efflux and ROS, as well as ATP release signaling through the $\mathrm{P} 2 \mathrm{X}_{7}$ receptor are also involved in caspase- 4 activation, perhaps through auto-catalytic cleavage, but this has not been investigated. While studies have investigated the role of caspases in Eh infection, none have addressed inflammatory caspase-4. Caspase-3 has been shown to be involved in Jurkat T cell apoptosis induced by $E h^{33}$ Additionally, using a general caspase inhibitor in Eh-challenged mice resulted in smaller liver abscesses. ${ }^{34}$ Furthermore, blocking $\mathrm{K}^{+}$channel activity and using specific inhibitors of caspase-1, -3 , and a pan-caspase inhibitor protected HT-29 intestinal epithelial cells and THP-1 macrophages from amebic killing. ${ }^{34}$ These studies underline the importance of inflammatory caspases in disease pathogenesis and its importance in alleviating Eh infection.

The NLRP3 inflammasome has been well-described as requiring the recruitment of ASC, NLRP3, and pro-caspase-1 into a high multimeric complex for the activation of caspase-1. Following this event, several pro-inflammatory cytokines such as IL-1 $\beta$ and IL-18 are cleaved into its bioactive form and secreted. However, it has always been an enigma whether inflammatory caspases could cross talk with each other. Eh-induced caspase- 4 activation and secretion was found to be independent of NLRP3 and ASC, indicating that there are alternate proteins that it interacts with or it can be auto-catalytically cleaved upon dimerization. Previous studies have shown that LPS can bind the CARD domain of caspase-4 causing homo-oligomerization and activation. ${ }^{31}$ Caspase-4 activation did not depend on caspase-1, indicating that further studies are warranted to assess how caspase-4 activation occurs. In our study, caspase-4-deficient macrophages stimulated with $E h$ for 60 min displayed less caspase-1 activation and IL-1 $\beta$ secretion compared to control THP- 1 macrophages, supporting our initial speculation that caspase- 4 and -1 interacted in this inflammatory cascade. A recent study found that caspase- 4 regulated caspase- 1 activity and IL-1 $\beta$ secretion in response to dengue infection, ${ }^{35}$ but did not address if regulation of $\mathrm{IL}-1 \beta$ secretion was due to direct caspase- 4 and -1 interactions. Other studies have suggested that caspase- 4 cannot cleave IL-1 $\beta^{36-38}$ but can directly bind to caspase- 1 and cleave it. ${ }^{39}$ Based on these observations we speculated that caspase- 1 could be a substrate for caspase-4. This was specifically a caspase- 4 and -1 interaction because overexpression of caspase- 4 in THP- 1 macrophages and overexpression of caspase- 4 , caspase- 1 , and IL-1 $\beta$ secretion in COS-7 cells, enhanced IL-1 $\beta$ release in response to $E h$. These findings implicate an important role for caspase- 4 in regulating caspase- 1 and this occurred by enhancing the cleavage of the CARD domain. The activation of caspase- 1 by caspase- 4 was likely mediated by the cysteine residue, which was shown to lose its proteolytic function when it was mutated. ${ }^{21}$ This phenomenon of caspase- 4 potentiating caspase- 1 activation in response to $E h$ is similar to MSU, as MSU did show a requirement for caspase-4 in IL$1 \beta$ release using CRISPR/Cas9 KO macrophages, similar to a previous study that used caspase-4 siRNA knockdown. ${ }^{21}$ Nigericin stimulation, however, did not require caspase-4 to enhance caspase- 1 activation. Our studies suggest that caspase- 4 enhancing effect is important in Eh infection, where live parasites upon contact with macrophages alert the immune system to activate several effector molecules to trigger an appropriate response. Interestingly, the mouse ortholog, caspase-11 also enhanced caspase-1 processing and $\mathrm{IL}-1 \beta$ production in response to Escherichia coli and cholera toxin B. ${ }^{40}$ However, in response to Eh, caspase-11 did not seem to play a role in mediating caspase-1 p20 activation. Caspase- 4 and -11 activation both occurred independently of NLRP3 and ASC and were not mediators of caspase- 1 activation, suggesting that they are not true orthologs. Caspase- 4 was found to be critical in mediating IL-1a and cell death in response to Gram-negative pathogens, Yersinia pseudotuberculosis, Salmonella enterica serovar Typhimurium, and Legionella pneumophila. ${ }^{14}$ Cell death was not observed to be dependent on caspase- 4 in response to $E h$ and neither did cell death differ among the caspase knockout macrophages.

As GSDMD is critical in inflammatory caspase-induced pyroptosis and IL-1 $\beta$ secretion, ${ }^{18,19,41}$ this pathway was investigated. GSDMD was found to be a key substrate for caspase- 4 and -1 mediated IL-1 $\beta$ secretion using CRISPR/Cas9 KO macrophages. In contrast to pyroptosis-dependent IL-1 $\beta$ secretion, "macrophage hyperactivation" was recently described for IL-1 $\beta$ secretion independent of cell death in response to Staphylococcus aureus. ${ }^{41}$ This is the same scenario with $E h$, where it also induces a state of hyperactivated macrophages leading to GSDMD-dependent IL-1 $\beta$ secretion in viable macrophages. In contrast to LPS and nigericin, it was shown to be a potent activator of caspase-1-GSDMDdependent IL-1 $\beta$ secretion consistent with previous studies. ${ }^{19}$ Interestingly, GSDMD-deficient macrophages displayed less caspase- 4 intermediate forms in the supernatant, but procaspase- 4 levels were the same compared to WT. GSDMD pores in the plasma membrane are $10-20 \mathrm{~nm}$ in diameter allowing for the passage of IL-1 $\beta(4 \mathrm{~nm}, 17 \mathrm{kDa})^{42}$ and caspase- 4 intermediate forms $(32-34 \mathrm{kDa})$. In response to $E h$, it appears that the primary function of GSDMD cleavage was not to trigger pyroptosis, but rather to act as a conduit for $\mathrm{IL}-1 \beta$ released as part of the proinflammatory response elicited by Eh. Pyroptosis was delayed as 
shown with the LDH assays and occurred after $1 \mathrm{~h}$. In addition to the $30 \mathrm{kDa} N$-terminal cleavage product of GSDMD, Eh-stimulated macrophages also induced the accumulation of a $26 \mathrm{kDa}$ cleavage product that was not present with LPS and nigericin stimulation. At present, we do not know the identity of this cleaved product but speculate that since $E h$ activates several caspases and proteases upon macrophage contact, other proteases could potentially cleave GSDMD at a site different from caspase- 4 and -1 .

Although there is no direct ligand for NLRP3, it is believed to sense commonly induced stimuli by a variety of agonizts. Caspase4 acts in a similar manner, where although there is no ligand, it is able to sense cellular perturbations to further activate caspase- 1 to regulate IL-1 $\beta$ secretion. Thus, IL- $1 \beta$ secretions are dictated by two checkpoints, where only the sensing of $E h$ via direct contact does it requires the aid of caspase- 4 to boost caspase- 1 activation. Caspase-1 activation alone, on the other hand, was sufficient to mount an immune response to nigericin. Covalent cross-linking with formaldehyde to stabilize caspase-4-caspase- 1 interactions and specific antibodies allowed us to detect the interacting caspases by western blot. We speculate that the interactions between the caspases are weak and transient based on the crosslinked complex when stimulated with $E h$ at different times. A schematic diagram depicting how Eh stimulates both caspase-4 and -1 independently to cleave GSDMD to regulate high output IL$1 \beta$ in macrophages is shown (Fig. 10).

Elucidating the inflammatory caspase-caspase interacting proteins will be important not only for understanding Eh pathogenesis, but also on how inflammation is regulated in infectious and inflammatory diseases. NLRP3 inflammasome and specifically caspase- 1 has been implicated in a variety of conditions including sepsis and inflammatory bowel disease. ${ }^{43,44}$ As current knowledge of caspase- 4 biology in inflammatory diseases is limited our study highlights a critical role of this protein in amplifying IL-1 $\beta$ secretion. Since caspase-4 plays an essential role in mediating caspase-1 bioactivity, it poses to be a novel therapeutic target for treating uncontrolled inflammation.

\section{MATERIALS AND METHODS}

\section{Cell preparation and stimulation}

THP-1 human monocytic cells (ATCC, Manassas, VA) were cultured in RPMI with $10 \%$ FBS, $10 \mathrm{mM}$ HEPES, $50 \mu \mathrm{M}$ 2-mercaptoethanol, $100 \mathrm{U} / \mathrm{ml}$ penicillin and $100 \mu \mathrm{g} / \mathrm{ml}$ streptomycin sulfate in a humidified incubator with $5 \% \mathrm{CO}_{2}$. THP-1 cells were plated onto 24-well plates at $4 \times 10^{5}$ cells/well with $50 \mathrm{ng} / \mathrm{mL}$ PMA (P1585, Sigma-Aldrich) in complete RPMI 1640 overnight. Cells were stimulated with Eh, EhCP-A5 ${ }^{-}$, or EhAP-A ${ }^{-}$in $250 \mu \mathrm{L}$ serum-free RPMI for the indicated times $(10,30$, and $60 \mathrm{~min})$. For inhibitor studies, THP- 1 cells were pretreated at $37^{\circ} \mathrm{C}$ unless otherwise stated with the indicated concentrations of DPI $(25-100 \mu \mathrm{M}, 1 \mathrm{~h}$ pretreatment, 300260, Calbiochem), $\mathrm{KCl}(90-200 \mathrm{mM}, 1 \mathrm{~h}$ pretreatment, P5405, Sigma-Aldrich), and oxidized ATP $(300 \mu \mathrm{M}, 2 \mathrm{~h}$ pretreatment, A6779, Sigma-Aldrich) before stimulation with Eh. All cells were changed to fresh serum-free RPMI prior to stimulation with Eh, LPS from E. coli 0111:B4 (L3012, SigmaAldrich), nigericin (N7143, Sigma-Aldrich), MSU (Y. Shi, Department of Immunology, University of Calgary, Canada), or ATP (A7699, Sigma-Aldrich).

THP-1 macrophages were stimulated with 1:20 Eh per well in $250 \mu \mathrm{L}$ serum-free RPMI for the indicated times. As a control for the silencing technology in $\mathrm{EhCP}-\mathrm{A} 5^{-}$we tested a vector control strain that had the same silencing technology as EhCP-A5 designated EhAP-A ${ }^{-}$. BMDM were cultured from bone marrow cells of C57BL/ 6 mice and incubated for 6 days in complete RPMI supplemented with $30 \%$ L929-cell supernatant. Cells were then plated at $5 \times 10^{5}$ cells/well in 24-well plates in complete RPMI 1640 . On the day of experiment, BMDM were treated with $1 \mu \mathrm{g} / \mathrm{ml}$ LPS for $3.5 \mathrm{~h}$ prior to stimulation with $E h$.

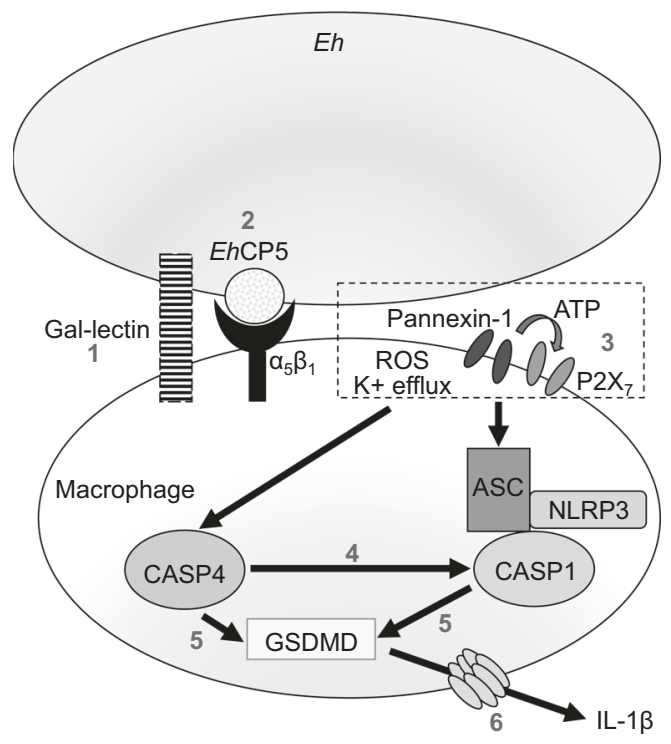

Fig. 10 Schematic representation of E. histolytica-macrophage interaction and induction of caspase- 4 and -1 activation and IL-1 $\beta$ release. The first step of caspase activation is $E h$ adherence to macrophage contact via the Gal-lectin adhesin that binds to Gal/ GalNAc residues on the macrophage surfaces (1). EhCP5 is a cysteine protease that is highly expressed on the surface of $E h$ and was shown to activate both caspase-4 and -1 (2). EhCP5 binds to $\alpha_{5} \beta_{1}$ integrin on macrophage surface to trigger ATP release through pannexin- 1 channel and subsequently signals back onto the $\mathrm{P} 2 \mathrm{X}_{7}$ receptor to activate the NLRP3 inflammasome. Simultaneously, $\mathrm{K}^{+}$ efflux and the generation of reactive oxygen species converge to activate the NLRP3 inflammasome and it also activates caspase-4 (3, boxed out). Caspase- 4 is simultaneously activated with caspase- 1 and caspase- 4 enhances caspase- 1 activity by cleaving the caspase- 1 CARD domains independent of the inflammasome complex (4). Both caspase- 4 and -1 cleaves GSDMD (5) to induce IL-1 $\beta$ release (6)

\section{E. histolytica culture}

E. histolytica HM-1:IMSS were grown axenically in TYI-S-3 medium with $100 \mathrm{U} / \mathrm{ml}$ penicillin and $100 \mu \mathrm{g} / \mathrm{mL}$ streptomycin sulfate at 37 ${ }^{\circ} \mathrm{C}$ in sealed $15 \mathrm{~mL}$ borosilicate glass tubes as described previously. ${ }^{45}$ To maintain virulence, trophozoites were regularly passed through gerbil livers as described. ${ }^{46}$ Ameba were harvested after $72 \mathrm{~h}$ of growth (log phase) by centrifugation at $200 \times g$ for $5 \mathrm{~min}$ at $4{ }^{\circ} \mathrm{C}$ and suspended in serum-free RPMI prior to stimulating cells. E. histolytica cultures deficient in EhCP-A5 and EhAP-A ${ }^{-}$(vector control) were a gift from D. Mirelman (Weizmann Institute of Science). To irreversibly inhibit cysteine protease activity, Eh were cultured overnight in E-64 $(100 \mu \mathrm{M}$, E3132, Sigma-Aldrich) as described previously. ${ }^{47}$

THP-1 defASC, NLRP3, CASP1, CASP4, and GSDMD CRISPR/Cas9 THP-1 KO cells

THP-1 defASC cells were purchased from Invivogen (thp-dasc). These cells were derived from THP-1 human monocytic cells that do not express ASC, but express native levels of NLRP3 and procaspase-1. NLRP3 CRISPR/Cas9 KO THP-1 cells were a gift from D. Muruve (Department of Immunology, University of Calgary, Canada). They used the gRNA CTGCAAGCTGGCCAGGTACCTGG and TGTCATAGCCCCGTAATCAACGG. CASP1 and CASP4 CRISPR/ Cas9 KO THP-1 cells were a gift from V. Hornung (Institute of Molecular Medicine, University Hospital, University of Bonn, Germany) and verified for CASP1 and CASP4 KO. They had used a plasmid encoding a CMV-mCherry-CAS9 expression cassette and a gRNA under the U6 promoter. The CRISPR target sites used were (PAM regions in bold): ATTGACTCCGTTATTCCGAAAGG (CASP1), GCTCATCCGAATATGGAGGCTGG (CASP4). Cells were maintained 
in complete RPMI media as described above. GSDMD CRISPR/Cas9 KO THP-1 cells were a gift from D. Bachovchin (Memorial Sloan Kettering Cancer Centre, New York, USA). The sgRNA used was TGAGTGTGGACCCTAACACC.

COS-7 cells

COS-7 cells were a gift from R. Yates (Department of Comparative Biology and Experimental Medicine, University of Calgary, Canada). COS-7 cells were transfected with caspase-4 (100 ng), caspase-1 $(10 \mathrm{ng})$, and pro-lL-1 $\beta(1 \mu \mathrm{g})$ plasmids $^{48}$ using jetPRIME transfection reagent (114-07, Polyplus) according to the manufacturer's protocol to determine protein interactions. The CASP4 plasmid was generated using the V51 pIRESpuro-GLUE (\#15100, Addgene) as the backbone vector. ${ }^{49}$ COS-7 cells were maintained using complete DMEM $(10 \%$ FBS, $100 \mathrm{U} / \mathrm{ml}$ penicillin and $100 \mu \mathrm{g} / \mathrm{ml}$ streptomycin sulfate) in a humidified incubator with $5 \% \mathrm{CO}_{2}$.

\section{Nucleofection}

Transfection of THP-1 cells was performed using the Nucleofector II ${ }^{\circledast}$ Device (AAD-1001S, Amaxa) according to the manufacturer's protocol with the Nucleofector V Kit (AMA-VCA1003) Nucleofector $^{\circledR}$ Program U-001 (THP-1 high viability program). Briefly, $1 \times 10^{6}$ cells were nucleofected with $1 \mu \mathrm{g}$ caspase- 4 plasmid and then PMA $(50 \mathrm{ng} / \mathrm{mL}$ ) differentiated overnight prior to experimentation the next day. Cells were replaced with serum-free media and then stimulated with Eh.

\section{Caspase-4 siRNA}

THP-1 cells were differentiated overnight with PMA $(50 \mathrm{ng} / \mathrm{mL})$ prior to transfection as described above. Cells were transfected with SMARTpool: SiGENOME caspase-4 siRNA (M-004404-01-0005, Dharmacon), or scramble siRNA (D-001206-14-05, Dharmacon) as a control, with the INTERFERin reagent (409-10, Polyplus), as per the manufacturer's protocol. A total of $10 \mu \mathrm{L}$ of INTERFERin reagent was used for each transfection with caspase-4 siRNA, at $50 \mathrm{nM}$, or scrambled siRNA as the control. Media was replaced with complete RPMI $24 \mathrm{~h}$ later. Eh stimulation was performed $48 \mathrm{~h}$ following siRNA transfection for 60 min by western blot and IL-1 $\beta$ secretion.

\section{Formaldehyde cross-link and co-immunoprecipitation}

Formaldehyde solution was obtained by dissolving $0.8 \%$ paraformaldehyde in PBS for $2 \mathrm{~h}$ at $\sim 80^{\circ} \mathrm{C}$. The solution was filtered $(0.22 \mu \mathrm{m})$ and stored in the dark at room temperature. THP-1 cells and COS-7 cells were stimulated with $E h$ for $20 \mathrm{~min}$. Cells were washed with $1 \mathrm{X}$ PBS and spun down at $300 \times g$ at $4^{\circ} \mathrm{C}$. Next, $0.8 \%$ formaldehyde was added at $1 \times 10^{7}$ cells $/ \mathrm{mL}$ for $7 \mathrm{~min}$ at room temperature and immediately quenched with ice-cold glycine to a final concentration of $125 \mathrm{mM}$. Sample was pelleted at $1800 \times g$ at room temperature for 3 min and supernatant was discarded. Cells were washed with glycine, transferred to a microtube, and spun down again at $300 \times g$ in $4^{\circ} \mathrm{C}$. Cell pellets were lysed in RIPA buffer at $1 \times 10^{8}$ cells $/ \mathrm{mL}$, along with the aid of a dounce homogenizer. Lysates were spun for $15 \mathrm{~min}$ at $18,000 \times g$ at $4{ }^{\circ} \mathrm{C}$ to remove insoluble debris. Caspase- 4 or caspase- 1 primary antibody was added at $1 \mu \mathrm{g}$ and placed on a shaker in $4{ }^{\circ} \mathrm{C}$ for $3 \mathrm{~h}$. Several different caspase- 4 antibodies were screened for use including: anti-caspase-4 (M029-3, MBL International Corporation), anticaspase-4 (NBP1-76602, Novus Biologicals), and anti-caspase-4 (\#4450, Cell Signaling). Several different caspase-1 antibodies were screened for use including: anti-caspase-1 (sc-2225, Santa Cruz), anti-caspase-1 (sc-56036, Santa Cruz), and anti-caspase-1 p20 (Bally-1, Adipogen). About $30 \mu \mathrm{L}$ of protein A/G PLUS-agarose beads (sc-2003, Santa Cruz) were added on a shaker at $4{ }^{\circ} \mathrm{C}$ overnight. The next day, the beads were spun down at $300 \times g$ at $4{ }^{\circ} \mathrm{C}$ and washed three times with RIPA buffer and 10 times with Tris, $\mathrm{NaCl}$, EDTA solution. The beads were then incubated with
$50 \mu \mathrm{L}$ of $1.5 \mathrm{X}$ sample buffer at $65^{\circ} \mathrm{C}$ for $5 \mathrm{~min}$. Samples were run on SDS-PAGE and probed with antibodies or stained with Coomassie Blue.

Immunoblot

THP-1 supernatants from four wells were pooled and centrifuged at $4{ }^{\circ} \mathrm{C}$ for $5 \mathrm{~min}$ at $2000 \times \mathrm{g}$. Pelleted debris was discarded and supernatants were concentrated by trichloroacetic acid (TCA) precipitation. For western blotting, precipitated supernatants were suspended in $50 \mu \mathrm{L}$ Laemmli buffer, boiled for $5 \mathrm{~min}$ and equal volumes were resolved on $12 \%$ polyacrylamide gels and transferred to nitrocellulose membranes. For cell lysates, plates were washed in cold 1X PBS before lysis buffer $(100 \mathrm{mM} \mathrm{NaCl}, 20$ $\mathrm{mM}$ Tris (pH 8), 0.1\% SDS, 0.5\% Triton X-100, 5 mM EDTA, $6.8 X$ PMSF, $0.1 \mathrm{mM} \mathrm{E}-64,1 \mu \mathrm{g} / \mathrm{L}$ leupeptin, $1 \mu \mathrm{g} / \mathrm{L}$ aprotonin, and $0.02 \%$ protease inhibitor cocktail (Sigma-Aldrich)) was added and centrifuged at $4^{\circ} \mathrm{C}$ for $15 \mathrm{~min}$ at $14,000 \times \mathrm{g}$. Protein concentrations were determined using the bicinchoninic acid (BCA) protein assay, using bovine serum albumin as a standard (\#23225, Thermo Scientific). Equal amounts of proteins boiled for $5 \mathrm{~min}$ in Laemmli buffer were resolved on $10-12 \%$ polyacrylamide gels and transferred to nitrocellulose membranes. Membranes were blocked in $5 \%$ skim milk, incubated overnight at $4{ }^{\circ} \mathrm{C}$ in primary antibodies and visualized with secondary HRP-conjugated antibodies. Supernatants were detected with SuperSignal Chemiluminescence Reagents (Pierce) and lysates with ChemiLucent ECL detection (EMD Millipore). Primary Abs were anti-IL-1 $\beta$ human (1:000, sc-7884, Santa Cruz), anti-caspase-4 human (1:1000, M0293, MBL International Corporation), anti-caspase-1 human (1:1000, sc-622, Santa Cruz), anti-caspase-1 human (1:1000, sc-56036, Santa Cruz), anti-GSDMD (1:1000, \#96458, Cell Signaling), anti-NLRP3 (1:1000, AG-20B-0014, Adipogen), anti-ASC (1:1000, sc-30153, Santa Cruz), anti-IL-1 $\beta$ mouse (1:000, AF-401, R\&D), anti-caspase11 mouse (1:1000, 17D9, Novus Biologicals), anti-caspase-1 p20 mouse (1:1000, Casper-1, Adipogen), and anti-GAPDH (1:10,000, Millipore, Sigma).

IL-1 $\beta$ assay in HEK-Blue ${ }^{\mathrm{TM}}$ reporter cells

Following stimulation of THP-1 macrophages, supernatants were kept on ice for immediate processing or frozen at $-80^{\circ} \mathrm{C}$. In the morning, HEK-Blue ${ }^{\mathrm{TM}}$ IL1 $\beta$ cells were plated in a 96-well plate at $4 \times 10^{5}$ cells $/ \mathrm{mL}$ with a total of $150 \mu \mathrm{L}$ in each well. Next, $50 \mu \mathrm{L}$ of supernatants from THP-1 macrophages were added undiluted into each well containing HEK-Blue ${ }^{\mathrm{TM}} \mathrm{IL} 1 \beta$ cells overnight at $37^{\circ} \mathrm{C}$ in an incubator with $5 \% \mathrm{CO}_{2}$. A standard curve using recombinant human IL-1 $\beta$ (200-01B, Peprotech) was made with serial dilutions (100 to $0.01 \mathrm{ng} / \mu \mathrm{L})$. A total of three replicates were performed for each treatment condition. The following day, $50 \mu \mathrm{L}$ of supernatant was transferred into a black 96 -well plate. Then $150 \mu \mathrm{L}$ of the QUANTI-Blue $^{\text {TM }}$ (rep-qb1, Invivogen) was added into each well. The QUANTI-Blue $^{\mathrm{TM}}$ is initially a pink color and eventually turns into blue over time, indicative of SEAP levels. The intensity of the color reaction is proportional to the amount of IL-1 $\beta$ in the supernatant from stimulated THP-1 macrophages. The plate was incubated at $37^{\circ} \mathrm{C}$ for $90 \mathrm{~min}$ and the SEAP levels were determined using a spectrophotometer at $655 \mathrm{~nm}$.

\section{Human IL-1 $\beta$ ELISA}

THP-1 macrophages were stimulated with Eh for the indicated times and the supernatants were centrifuged to remove cellular components and this was used directly for enzyme-linked immunosorbent assay (ELISA, DY201, R\&D) as per the manufacturer's protocol.

Human focused 13-plex cytokine/chemokine array Cell supernatants of stimulated THP-1 macrophages were also analyzed for the presence of other cytokines and chemokines. This sensitive luminex human focused 13-plex-discovery assay was 
performed by Eve Technologies (Calgary, AB) that looked at the following cytokines/chemokines: GM-CSF, IFN- $\gamma$, IL-1 $\beta$, IL-2, IL-4, IL-6, IL-8, IL-10, IL-12, MCP-1, TNF-a, IL-13, and IL-5.

Lactate dehydrogenase assay

LDH levels were measured using the Promega ${ }^{\mathrm{TM}}$ CytoTox-ONE homogeneous membrane integrity assay (G7890, Fisher Scientific) as per the manufacturer's protocol. Relative LDH release was calculated using the equation: $\mathrm{LDH}$ (\% release) $=\%$ of ((LDH released from stimulation-background)/(maximum $\mathrm{LDH}$ releasedbackground)). The LDH (\% release from control) was calculated using the $\mathrm{LDH}$ (\% release) subtracted from non-stimulated cells.

\section{Animals}

Male C57BL/6 mice, 8-10 weeks old, were purchased from Charles River. Casp $1 / 11^{-/-}$mice, bred onto a C57BL/6 background, were obtained from Dr. Yan Shi (University of Calgary). Nlrp3 ${ }^{-1-}$ and $\mathrm{Asc}^{-1-}$ mice bred onto a C57BL/6 background, were obtained from Dr. Dan Muruve. Casp $11^{-/-}$femurs and tibia were obtained from Dr. Bruce Vallance (Department of Pediatrics, University of British Columbia). Femurs and tibia from these mice were used for the growth of BMDM.

\section{Statistics}

All experiments shown are representative of three independent experiments unless otherwise indicated. GraphPad Prism 5 (Graph-Pad Software, San Diego, CA) was used for statistical analysis. Treatment groups were compared using the paired Student's $t$-test or analysis of variance (ANOVA) with a Bonferroni post-hoc test. Statistical significance was assumed at $p<0.05$. Results are displayed as mean $+/$-standard error of the mean (SEM).

\section{ACKNOWLEDGEMENTS}

We thank Y. Shi for the MSU, Casp1/11 $1^{-1}$ mice, D. Muruve for the NLRP3 CRISPR KO cells, NIrp3 ${ }^{-1-}$ and $\mathrm{Asc}^{-1-}$ mice, V. Hornung for the CASP1 and CASP4 CRISPR KO cells, D. Bachovchin for the GSDMD CRISPR KO cells, and R. Yates for the HEK-Blue ${ }^{\mathrm{TM}} \mathrm{IL}-1 \beta$ cells and COS-7 cells. This work was funded by a Discovery Grant (RGPIN-2014-04023) from the Natural Sciences and Engineering Research Council of Canada awarded to K. C. The funders had no role in study design, data collection and analysis, decision to publish, or preparation of the manuscript.

\section{AUTHOR CONTRIBUTIONS}

J.Q. and K.C. were responsible for experimental design. K.C. and F.M. contributed reagents and methodical support. J.Q. performed the majority of experiments and analysis of data. F.M. performed the nucleofection experiments and the formaldehyde cross-linking. C.S. generated the NLRP3 CRISPR/Cas9 KO THP-1 macrophages. J.Q.and K.C. wrote the manuscript.

\section{ADDITIONAL INFORMATION}

The online version of this article (https://doi.org/10.1038/s41385-018-0101-9) contains supplementary material, which is available to authorized users.

Competing interests: The authors declare no competing interests.

Ethics statement: The Health Sciences Animal Care Committee from the University of Calgary, have examined the animal care and treatment protocol (AC14-0219) and approved the experimental procedures proposed and certifies with the applicant that the care and treatment of animals used was in accordance with the principles outlined in the most recent policies on the "Guide to the Care and Use of Experimental Animals" by The Canadian Council on Animal Care.

\section{REFERENCES}

1. WHO. Amebiasis. Wkly. Epidemiol. Rec. 7, 97-100 (1997)

2. Espinosa-Cantellano, M. \& Martínez-Palomo, A. Pathogenesis of intestinal amebiasis: from molecules to disease. Clin. Microbiol. Rev. 13, 318-331 (2000).
3. Duggal, P. et al. A mutation in the leptin receptor is associated with Entamoeba histolytica infection in children. J. Clin. Invest. 121, 1191-1198 (2011).

4. Mondal, D., Haque, R., Sack, R. B., Kirkpatrick, B. D. \& Petri, W. A. Short report: attribution of malnutrition to cause-specific diarrheal illness: evidence from a prospective study of preschool children in Mirpur, Dhaka, Bangladesh. Am. J. Trop. Med. 80, 824-826 (2009).

5. Lin, J. Y. \& Chadee, K. Macrophage cytotoxicity against Entamoeba histolytica trophozoites is mediated by nitric oxide from L-arginine. J. Immunol. 148, 3999-4005 (1992).

6. Petri, W. A., Smith, R. D., Schlesinger, P. H., Murphy, C. F. \& Ravdin, J. I. Isolation of the galactose-binding lectin that mediates the in vitro adherence of Entamoeba histolytica. J. Clin. Invest. 80, 1238-1244 (1987).

7. Ravdin, J. I., John, J. E., Johnston, L. I., Innes, D. J. \& Guerrant, R. L. Adherence of Entamoeba histolytica trophozoites to rat and human colonic mucosa. Infect. Immun. 48, 292-297 (1985).

8. St-Pierre, J. et al. The macrophage cytoskeleton acts as a contact sensor upon interaction with Entamoeba histolytica to trigger IL-1 $\beta$ secretion. PLoS. Pathog. 13. e1006592 (2017).

9. Mortimer, L., Moreau, F., Cornick, S. \& Chadee, K. The NLRP3 inflammasome is a pathogen sensor for invasive Entamoeba histolytica via activation of a5 $\beta 1$ integrin at the macrophage-amebae intercellular junction. PLoS. Pathog. 11, e1004887 (2015).

10. Mortimer, L., Moreau, F., Cornick, S. \& Chadee, K. Gal-lectin-dependent contact activates the inflammasome by invasive Entamoeba histolytica. Mucosal Immunol. 7, 829-841 (2013).

11. Martinon, F., Mayor, A. \& Tschopp, J. The inflammasomes: guardians of the body. Annu. Rev. Immunol. 27, 229-265 (2009).

12. Knodler, L. A. et al. Non-canonical inflammasome activation of caspase-4/caspase-11 mediates epithelial defenses against enteric bacterial pathogens. Cell. Host. Microbe 16, 249-256 (2015).

13. Lakshmanan, U. \& Porter, A. G. Caspase-4 interacts with TNF receptor-associated factor 6 and mediates lipopolysaccharide-induced NF-KB-dependent production of IL-8 and CC chemokine ligand 4 (macrophage-inflammatory protein-1). J. Immunol. 179, 8480-8490 (2007).

14. Casson, C. N. et al. Human caspase-4 mediates noncanonical inflammasome activation against gram-negative bacterial pathogens. Proc. Natl Acad. Sci. USA 112, 6688-6693 (2015).

15. Wang, S. et al. Murine caspase-11, an ICE-interacting protease, is essential for the activation of ICE. Cell 92, 501-509 (1998).

16. Wang, S. Y. et al. Identification and characterization of Ich-3, a member of the interleukin-1 $\beta$ converting enzyme (ICE) ced-3 family and an upstream regulator of ICE. J. Biol. Chem. 271, 20580-20587 (1996).

17. Kayagaki, N. et al. Caspase-11 cleaves gasdermin D for non-canonical inflammasome signalling. Nature 526, 666-671 (2015).

18. Shi, J. et al. Cleavage of GSDMD by inflammatory caspases determines pyroptotic cell death. Nature 526, 660-665 (2015)

19. He, W. T. et al. Gasdermin D is an executor of pyroptosis and required for interleukin-1 $\beta$ secretion. Cell Res. 25, 1285-1298 (2015).

20. Hornung, V. et al. Silica crystals and aluminum salts activate the NALP3 inflammasome through phagosomal destabilization. Nat. Immunol. 9, 847-856 (2008).

21. Sollberger, G., Strittmatter, G. E., Kistowska, M., French, L. E. \& Beer, H.-D. Caspase4 is required for activation of inflammasomes. J. Immunol. 188, 1992-2000 (2012).

22. Zhang, Z. et al. Entamoeba histolytica cysteine proteinases with interleukin-1 beta converting enzyme (ICE) activity cause intestinal inflammation and tissue damage in amoebiasis. Mol. Microbiol 37, 542-548 (2000).

23. Moncada, D., Keller, K. \& Chadee, K. Entamoeba histolytica cysteine proteinases disrupt the polymeric structure of colonic mucin and alter its protective function. Infect. Immun. 71, 838-844 (2003).

24. Lidell, M. E., Moncada, D. M., Chadee, K. \& Hansson, G. C. Entamoeba histolytica cysteine proteases cleave the MUC2 mucin in its C-terminal domain and dissolve the protective colonic mucus gel. Proc. Natl Acad. Sci. USA 103, 9298-9303 (2006).

25. Schmid-burgk, J. L. et al. Caspase-4 mediates non-canonical activation of the NLRP3 inflammasome in human myeloid cells. Eur. J. Immunol. 45, 2911-2917 (2015).

26. Baker, P. J. et al. NLRP3 inflammasome activation downstream of cytoplasmic LPS recognition by both caspase-4 and caspase-5. Eur. J. Immunol. 45, 2918-2926 (2015).

27. Broz, P. \& Monack, D. M. Noncanonical inflammasomes: caspase-11 activation and effector mechanisms. PLoS. Pathog. 9, 9-12 (2013).

28. Pop, C. \& Salvesen, G. S. Human caspases: activation, specificity, and regulation. J. Biol. Chem. 284, 21777-21781 (2009).

29. Yang, J. et al. Mechanism of gasdermin D recognition by inflammatory caspases and their inhibition by a gasdermin D-derived peptide inhibitor. Proc. Natl Acad. Sci. USA 115, 6792-6797 (2018). 
30. Kast, J. \& Klockenbusch, C. Optimization of formaldehyde cross-linking for protein interaction analysis of non-tagged integrin $\beta 1$. J. Biomed. Biotechnol. 2010, 1-13 (2010).

31. Shi, J. et al. Inflammatory caspases are innate immune receptors for intracellular LPS. Nature 514, 187-192 (2014).

32. Muñoz-Planillo, R. et al. K+efflux is the common trigger of NLRP3 inflammasome activation by bacterial toxins and particulate matter. Immunity 38, 1142-1153 (2013).

33. Huston, C. D., Houpt, E. R., Mann, B. J., Hahn, C. S. \& Petri, W. A. Caspase 3dependent killing of host cells by the parasite Entamoeba histolytica. Cell Microbiol. 2, 617-625 (2000).

34. Yan, L. \& Stanley, S. L. Blockade of caspases inhibits amebic liver abscess formation in a mouse model of disease. Infect. Immun. 69, 7911-7914 (2001).

35. Cheung, K. T., Sze, D. Myuen, Chan, K. H. \& Leung, P. H. Involvement of caspase-4 in IL-1 beta production and pyroptosis in human macrophages during dengue virus infection. Immunobiology 223, 356-364 (2017).

36. Faucheu, $C$. et al. A novel human protease similar to the interleukin- $1 \beta$ converting enzyme induces apoptosis in transfected cells. EMBO J. 14, 1914-1922 (1995).

37. Munday, N. A. et al. Molecular cloning and pro-apoptotic activity of ICErelll and ICErellII, members of the ICE/CED-3 family of cysteine proteases. J. Biol. Chem. 270, 15870-15876 (1995)

38. Kamens, J. et al. Identification and characterization of $\mathrm{ICH}-2$, a novel member of the interleukin-1 $\beta$-converting enzyme family of cysteine proteases. J. Biol. Chem. 270, 15250-15256 (1995).

39. Kajiwara, Y. et al. A critical role for human caspase-4 in endotoxin sensitivity. J. Immunol. 193, 335-343 (2014).
40. Kayagaki, N. et al. Non-canonical inflammasome activation targets caspase- 11 . Nature 479, 117-121 (2011).

41. Evavold, C. L. et al. The pore-forming protein gasdermin D regulates interleukin-1 secretion from living macrophages. Immunity 48, 35-44 (2017).

42. Heilig, R. et al. The gasdermin-D pore acts as a conduit for IL-1 $\beta$ secretion in mice. Eur. J. Immunol. 48, 584-592 (2018).

43. Scott, A. \& Saleh, M. The inflammatory caspases: guardians against infections and sepsis. Cell Death Differ. 14, 23-31 (2007).

44. Becker, C., Watson, A. J. \& Neurath, M. F. Complex roles of caspases in the pathogenesis of inflammatory bowel disease. Gastroenterology 144, 283-293 (2013).

45. Diamond, L. S., Harlow, D. A. N. R. \& Cunnick, C. C. A new medium for the axenic cultivation of Entamoeba and other Entamoeba histolytica. Trans. R. Soc. Trop. Med. Hyg. 72, 431-432 (1978).

46. Denis, M. \& Chadee, K. Cytokine activation of murine macrophages for in vitro killing of Entamoeba histolytica trophozoites. Infect. Immun. 57, 1750-1756 (1989).

47. Hou, Y., Mortimer, L. \& Chadee, K. Entamoeba histolytica cysteine proteinase 5 binds integrin on colonic cells and stimulates $\mathrm{NF}_{\mathrm{K}} \mathrm{B}$-mediated pro-inflammatory responses. J. Biol. Chem. 285, 35497-35504 (2010).

48. Mortimer, L., Moreau, F., MacDonald, J. A. \& Chadee, K. NLRP3 inflammasome inhibition is disrupted in a group of auto-inflammatory disease CAPS mutations. Nat. Immunol. 17, 1176-1186 (2016).

49. Angers, S. et al. The KLHL12-Cullin-3 ubiquitin ligase negatively regulates the Wnt- $\beta$-catenin pathway by targeting dishevelled for degradation. Nat. Cell Biol. 8, 348-357 (2006). 